\title{
Thoracic dysfunction in whiplash associated disorders: A systematic review
}

\author{
Nicola R. Heneghan ${ }^{1}$, Richard Smith², Isaak Tyros ${ }^{3}$, Deborah Falla ${ }^{1}$, Alison Rushton ${ }^{1}$ \\ 1 Centre of Precision Rehabilitation for Spinal Pain, School of Sport, Exercise \& Rehabilitation Sciences, \\ University of Birmingham, Birmingham, United Kingdom, 2 Department of Allied Health Professions, \\ University of the West of England, Bristol, United Kingdom, 3 Edgbaston Physiotherapy Clinic, Birmingham, \\ United Kingdom \\ *n.heneghan@bham.ac.uk
}

\section{Abstract}

\section{Background}

Research investigating Whiplash Associated Disorder (WAD) has largely focused on the cervical spine yet symptoms can be widespread. Thoracic spine pain prevalence is reported $\sim 66 \%$; perhaps unsurprising given the forceful stretch/eccentric loading of posterior structures of the spine, and the thoracic spine's contribution to neck mobility/function. Approximately 50\% WAD patients develop chronic pain and disability resulting in high levels of societal and healthcare costs. It is time to look beyond the cervical spine to fully understand anatomical dysfunction in WAD and provide new directions for clinical practice and research.

Citation: Heneghan NR, Smith R, Tyros I, Falla D, Rushton A (2018) Thoracic dysfunction in whiplash associated disorders: A systematic review. PLoS ONE 13(3): e0194235. https://doi. org/10.1371/journal.pone.0194235

Editor: Pei-Ning Wang, Taipei Veterans General Hospital, TAIWAN

Received: January 14, 2017

Accepted: February 27, 2018

Published: March 23, 2018

Copyright: ๑ 2018 Heneghan et al. This is an open access article distributed under the terms of the Creative Commons Attribution License, which permits unrestricted use, distribution, and reproduction in any medium, provided the original author and source are credited.

Data Availability Statement: All relevant data are within the paper and its Supporting Information files.

Funding: The authors received no specific funding for this work.

Competing interests: The authors have declared that no competing interests exist.

\section{Purpose}

To evaluate the scope and nature of dysfunction in the thoracic region in patients with WAD.

\section{Methods}

A systematic review and data synthesis was conducted according to a pre-defined, registered (PROSPERO, CRD42015026983) and published protocol. All forms of observational study were included. A sensitive topic-based search strategy was designed from inception to $1 / 06 / 16$. Databases, grey literature and registers were searched using a study population terms and key words derived from scoping search. Two reviewers independently searched information sources, assessed studies for inclusion, extracted data and assessed risk of bias. A third reviewer checked for consistency and clarity. Extracted data included summary data: sample size and characteristics, outcomes, and timescales to reflect disorder state. Risk of bias was assessed using the Newcastle-Ottawa Scale. Data were tabulated to allow enabling a semi-qualitative comparison and grouped by outcome across studies. Strength of the overall body of evidence was assessed using a modified GRADE.

\section{Results}

Thirty eight studies $(n>50,000)$ which were conducted across a range of countries were included. Few authors responded to requests for further data ( 5 of 9 contacted). Results 
were reported in the context of overall quality and were presented for measures of pain or dysfunction and presented, where possible, according to WAD severity and time point post injury. Key findings include: 1) high prevalence of thoracic pain ( $>60 \%)$; higher for those with more severe presentations and in the acute stage, 2) low prevalence of chest pain $(<22 \%)$, 3) evidence of thoracic outlet syndrome, with some association to and involvement of the brachial plexus, 4) muscle dysfunction in the form of heightened activity of the sternocleidomastoid or delayed onset of action of the serratus anterior, 5) high prevalence of myofascial pain and trigger points in the scalene muscles, sternocleidomastoid and mid/lower fibres of trapezius muscle (48-65\%), and 6) inconclusive evidence of altered thoracic posture or mobility.

\section{Conclusions}

Considerable evidence supports thoracic pain and dysfunction in patients with WAD, involving primarily nerves and muscles. Notwithstanding the low/very low level of evidence from this review, our findings do support a more extensive clinical evaluation of patients presenting with WAD. Additional high quality research is required to further characterise dysfunction across other structures in the thoracic region, including but not limited to the thoracic spine (mobility and posture) and thoracic muscles (stiffness, activation patterns). In turn this may inform the design of clinical trials targeting such dysfunction.

\section{Introduction}

The cumulative incidence of patients seeking healthcare post-whiplash from a road traffic accident has increased over the last 30 years to an annual incidence of between 3 and 6/1000 inhabitants in North America and Western Europe [1]. Following injury, individuals experience a range of clinical manifestations, described as Whiplash Associated Disorder (WAD), including neck pain, fatigue, nausea, low self-reported physical and mental health, cognitive impairments and pain in multiple sites [2]. The severity of presentation in WAD is categorised according to the Quebec Task Force Classification (QTF) where the presence of clinical signs and symptoms relate to the severity of the disorder [3].

Whilst research has identified risk factors for poor prognosis $[4,5]$, and enhanced understanding of neurophysiological changes [6], it is not known why disability and pain persist beyond normal tissue healing times. With $40-60 \%$ patients progressing to experience chronic whiplash associated disorder (CWAD), estimated costs of $\sim \$ 4$ billion (USA) and $\sim 10$ billion (Europe) associated with management and time off work [7,8], further research is needed to fully understand anatomical dysfunction in WAD and provide new directions for clinical practice and research. This includes the effects on anatomically related body regions, such as the thoracic spine. Potential ongoing dysfunction in the thoracic region may partially explain why there is inconclusive evidence for the effectiveness of physiotherapy management for WAD II, where interventions target a primary complaint of neck pain $[9,10]$.

Although current research into WAD has focused on the primary complaint of neck pain [11], symptoms may also include stiffness $[12,13]$ and pain in other regions including the jaw, head, upper and lower limbs, chest, abdomen and groin [14]. Moreover, data from a large cohort study $(n=6481)$ reported that $66 \%$ of individuals complained of thoracic mid-spine pain post whiplash injury, with $23 \%$ still experiencing symptoms one year later [14]. This is 
not surprising given the mechanism of a whiplash injury which involves forceful stretch loading to the upper back muscles; muscles which span both the neck and thoracic spine [15]. Recent evidence supports the presence of pathology in the mid/lower fibres of the trapezius muscle where they insert onto bone (myofascial-entheseal dysfunction) [16], which may partly account for the high prevalence of thoracic pain reported in both acute (65.5\%) [14] and $>80 \%$ in chronic WAD [17]. Furthermore, a number of abnormalities have been documented for the trapezius muscle in people with chronic WAD including histological changes as well as changes in muscle behaviour $[18,19]$.

With reduced cervical mobility being characteristic of chronic WAD and evidence that the thoracic spine contributes up to $33 \%$ and $21 \%$ of the movement occurring during neck flexion and rotation respectively [20] perhaps thoracic mobility is impaired following a whiplash injury; however at this time relatively little is known about the impact of WAD on this spinal region [21]. Research is now needed to examine the impact of injury on the thoracic spine in WAD.

Nevertheless, a systematic review of the current evidence has never been conducted to examine the scope and nature of dysfunction/impairment in the thoracic spine region following whiplash injury and in WAD. Knowledge of such dysfunction may be used to inform clinical practice and examination of patients with WAD, but also future clinical trials of novel interventions targeting thoracic impairments in WAD.

\section{Objectives}

The primary objective is to evaluate the scope and nature of dysfunction in the thoracic spine region in patients with WAD. A secondary objective is to explore the scope and nature of such changes based on severity using the Quebec Task Force classification (I-III) and stage post injury (acute/sub-acute less than 3 months or chronic 3+ months). Thirdly, we wish to make evidence based recommendations for clinical practice and future research.

\section{Methods/Design}

\section{Protocol and registration}

A systematic review of all forms of observational study was conducted according to a predefined protocol [22], in line with the Centre of Research and Dissemination Guidelines [23], Meta-analyses of Observational Studies in Epidemiology (MOOSE) [24] and is reported in line with Preferred Reporting Items for Systematic Reviews and Meta-Analyses (PRISMA) [25], S1 Table. PROSPERO (Registration number: CRD42015026983).

\section{Eligibility criteria}

Eligibility criteria informed using SPIDER [26], included that the sample (S) comprised patients aged $>19$ years; the phenomenon of interest (PI) was a WAD following motor vehicle or sporting injury; investigated using an observational study design (cohort, case control, single case study) (D) with evaluation of patient reported or performance based measure(s) of thoracic dysfunction of one or more of the following: muscle with an insertion to the thoracic cage, bone or joint of the thoracic cage, neural tissue related to the thorax (E).

Exclusion criteria included: studies investigating upper trapezius, studies investigating a central pain mechanism or neurophysiology of pain where no testing took place in the thoracic region, simulation or modelling studies, fractures (WAD IV), visceral injury or fibromyalgia. 


\section{Information sources}

The search employed sensitive topic-based strategies designed for each database from inception to $1 / 6 / 16$. No language or geographical restrictions were included. Databases included, CINAHL, EMBASE, MEDLINE, ZETOC, Index to Chiropractic Literature ChiroAccess and Google Scholar. Selected Internet sites and Indexes including, Turning Research into Practice, PubMed, National Research Register and Cochrane Back Review Group were also searched. Hand searching of key journals included Spine and the European Spine Journal. Grey literature included British National Bibliography for Report Literature, Dissertation Abstracts, Index to Scientific and Technical Proceedings, National Technical Information Service and the System for Information on Grey Literature.

\section{Search strategy}

The search strategy included terms related to whiplash associated disorder and patient reported or performance based measures of thoracic dysfunction. Terms and keywords derived from the scoping search and experts [subject specific (NRH,AR) and methodological $(\mathrm{NRH}, \mathrm{AR})]$ included: 'whiplash', 'whiplash associated disorder', 'WAD', 'whiplash injury', 'motor vehicle accident OR collision', 'road traffic accident', 'cervical strain' and 'thoracic spine', 'dorsal spine', 'mid-spine', 'thoracic injuries', limiting to adults $>19$ years and diagnosis to achieve the best balance of sensitivity and specificity. An example a search from Medline is included S2 Table. Terms were adapted to reflect spelling differences and [14] unique searching features of individual databases. Reference lists of included papers were also searched.

\section{Study selection}

Two reviewers (NRH, RS) independently searched information sources and assessed identified studies for inclusion, facilitated by grading each eligibility criterion as eligible/not eligible/ might be eligible [27]. Full texts were reviewed and included when both reviewers agreed [23]. A third reviewer (IT) mediated in the event of disagreement [28].

\section{Data collection process and items}

Using a standardised form, the two reviewers extracted data independently [23]. A further reviewer (IT) independently examined data for accuracy and clarity. Authors were contacted for additional information or data where required.

\section{Data items}

Data were extracted from each study, including: study design, sample characteristics including age, gender, severity of WAD using the QTF Classification if reported, time point post injury and patient reported or performance based measures of thoracic dysfunction.

\section{Risk of bias in individual studies}

Risk of bias for each included study was independently assessed by the same initial reviewers. The third reviewer mediated in situations of disagreement. All tools and processes were piloted prior to use. Risk of bias was only assessed for cohort and case-control studies using the Newcastle-Ottawa Scale [29]; that includes eight items that are rated and categorised into three groups, namely selection, comparability and outcome. 


\section{Summary measures}

Summary measures of patient reported or performance based measures of thoracic dysfunction are presented in the form of prevalence data and confidence intervals where provided, for thoracic pain, chest pain, thoracic outlet syndrome (TOS), myofascial pain and trigger points, dysfunction involving the brachial plexus, thoracic spine posture or mobility. Results are presented where possible, according to severity (QTF) and stage of WAD i.e. acute/sub-acute $(<3$ months) or chronic (> 3 months).

\section{Synthesis of results}

In accordance with the protocol [22] meta-analyses would be performed where a sufficient number of studies share all of the stated characteristics; design, measure of dysfunction, severity based on QTF and stage post whiplash injury.

\section{Quality of evidence across studies}

Quality of evidence, including risk of bias across studies was evaluated using GRADE [30] for individual outcomes of interest. By their very nature, observational studies are considered 'low quality' although could be upgraded where a large dose response was evident, or the effects could not be accounted for by bias [30]. Likewise, findings could be downgraded to 'very low' where concerns were identified from the body of studies relating to precision, consistency, directness, precision or potentially other additional domains relating to strength of association e.g. magnitude of effect [30].

\section{Results}

\section{Study selection}

A total of 38 studies met the eligibility criteria, including 19 cohort studies, 16 case control studies and 3 single case studies/reports. The process of selection is detailed in Fig 1, with the list of excluded studies and reasons provided S3 Table.

\section{Study characteristics}

Study characteristics are detailed in Table 1. A range of measures of thoracic dysfunction were identified, including thoracic pain, chest pain, involvement of the brachial plexus, thoracic outlet syndrome, changes in muscle activation etc. An overview of the types of dysfunction is provided in Table 2 and, where possible, is presented according to severity and stage following injury.

\section{Risk of bias within studies}

Agreement of risk of bias following discussion was excellent with studies ranging considerably in quality from $1 / 9$ to $7 / 9$. Key methodological flaws included poor definition of cases, representativeness of cases, lack of non-exposed cohort, lack of blinding, controlling for risk factors beyond age and gender and clarity of follow up time point in cohort studies. There were many instances where information was missing and email requests for additional data or clarification was unforthcoming, resulting in ratings being 'unclear'. See Table 3 for risk of bias for cohort studies and Table 4 for case control studies. 


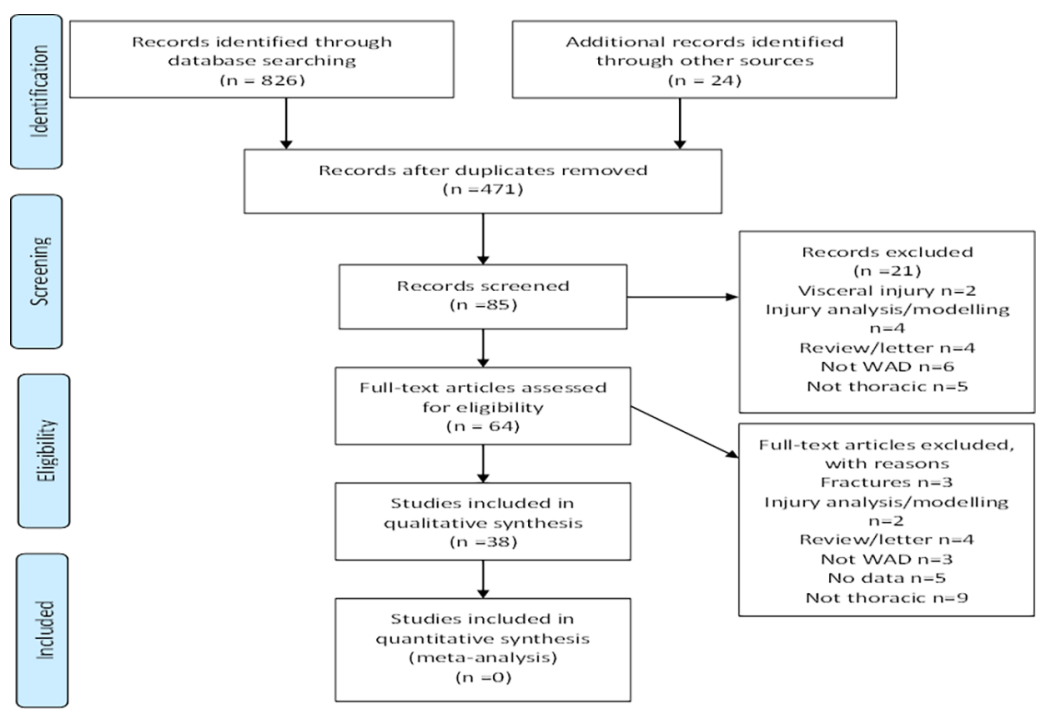

Fig 1. Flow chart of search and study selection.

https://doi.org/10.1371/journal.pone.0194235.g001

\section{Results of individual studies}

From this review we identified evidence of the following dysfunction,: thoracic spine pain in acute/sub-acute/chronic WAD ranging from minor injuries to more severe (WADIII) presentations $[11,14,17,32,33,43,50,52,58,62]$; chest pain in acute/sub-acute/chronic WAD [11, $17,52,53]$; postural changes [44, 61] and reduced chest/thoracic mobility in CWAD [61]; thoracic outlet syndrome in $\operatorname{CWAD}[31,35,36,47,51]$; involvement of the brachial plexus at all stages and across all levels of WAD severity [12, 46, 55, 57, 59]; muscle dysfunction in the form of the following: 1) heightened activity of the sternocleidomastoid during neck flexion [12, 56], 2) delayed onset of serratus anterior during arm elevation at the chronic stage in mild WAD [45] and 3) a high prevalence of myofascial pain and trigger points in the scalene muscles [40, 41], sternocleidomastoid [37, 40, 41] and mid/lower fibres of trapezius [16] within the subacute and chronic stages and across different levels of severity.

\section{Synthesis of results}

Synthesis of findings from cohort and case control studies across outcomes of dysfunction are provided in Tables 5-8. Sub-grouping according to stages and defined populations are included where reported. A summary of overall quality is provided based on GRADE following appraisal of risk of bias, consistency, precision, directness and effect size. Clinical heterogeneity across samples with respect to severity of presentation, time post injury and outcomes precluded meta-analysis being performed. Few studies stated a primary aim which accurately reflected the aims and objectives of this review.

Thoracic spine pain. Despite the very low quality of included studies, there is evidence of thoracic spine pain in a sub-acute WAD population $(\mathrm{n}=11,576)[11,14,17,34,39,43,52,60]$, with prevalence ranging between $21 \%-66 \%$. Findings were inconsistent in chronic WAD, with prevalence ranging $0-94 \%[17,50]$. Study quality, differing time points post injury, differing measurement approaches and higher degrees of WAD severity could partly account for the inconsistency. For example, chronic WAD, studies with less severe presentations (minor or WADII) $[32,50]$ demonstrated lower prevalence rates $(0-15.3 \%)$ compared to studies investigating WADII/III where prevalence was 54\% [58]. Just one study reporting thoracic pain 


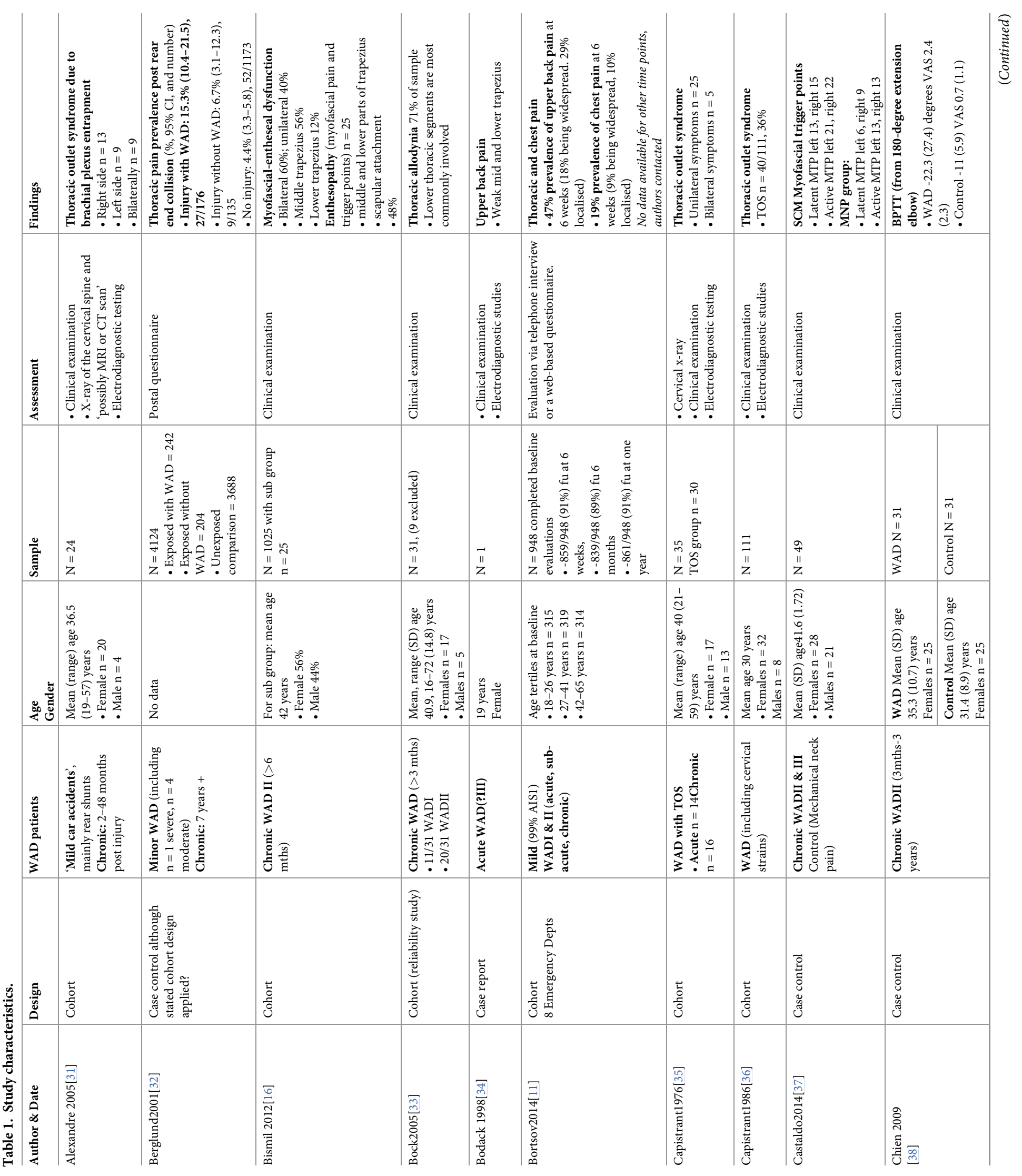




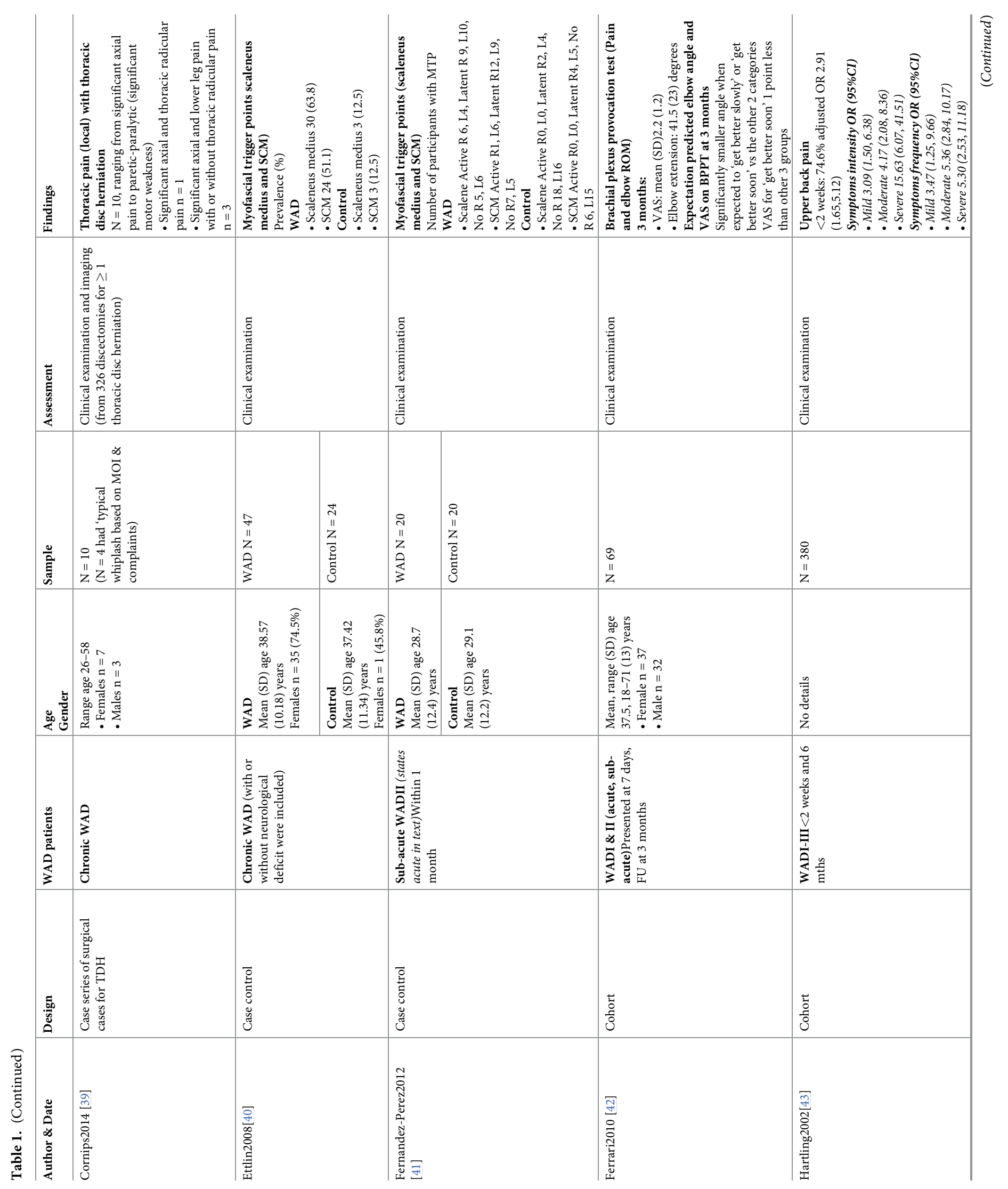




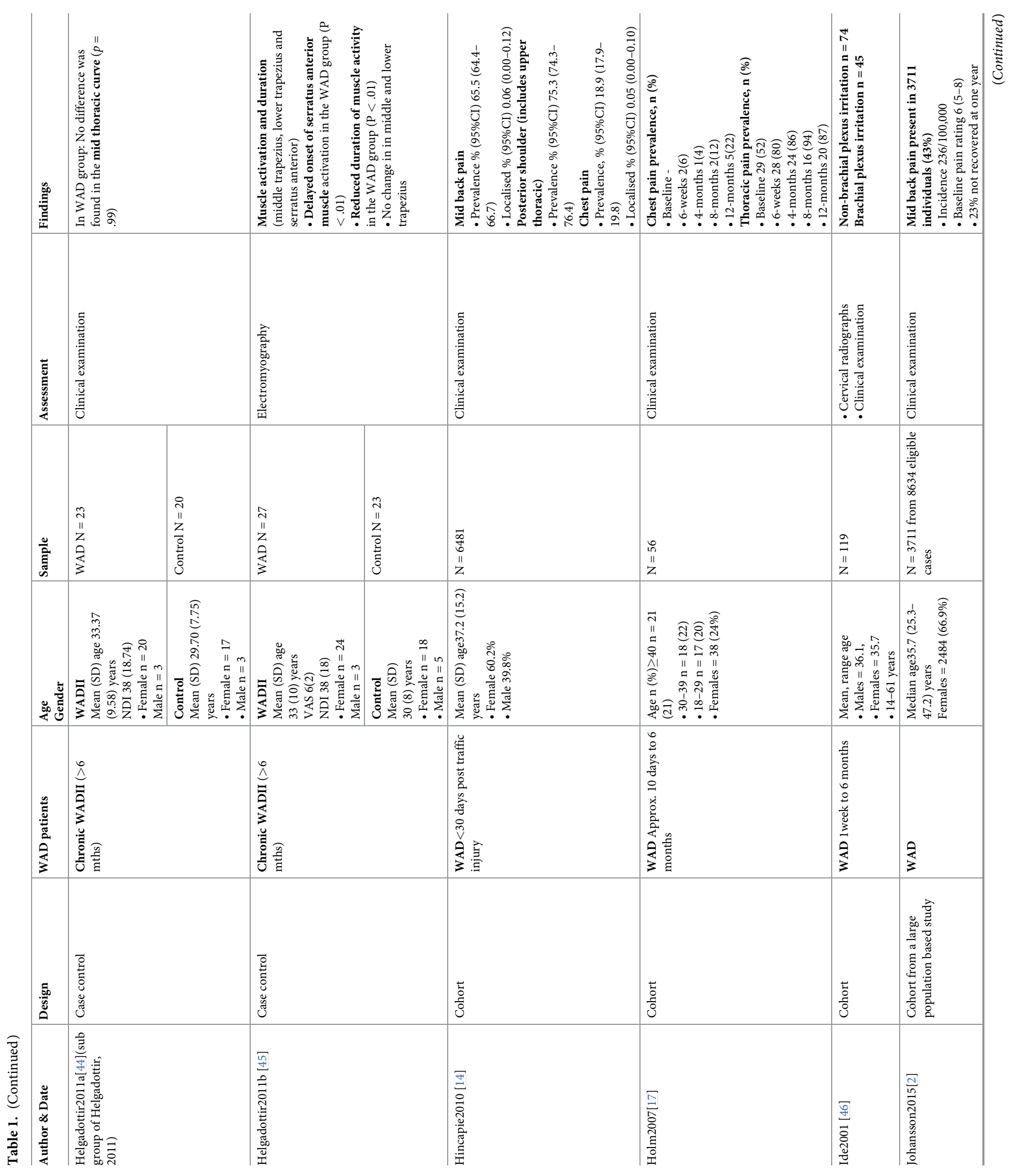




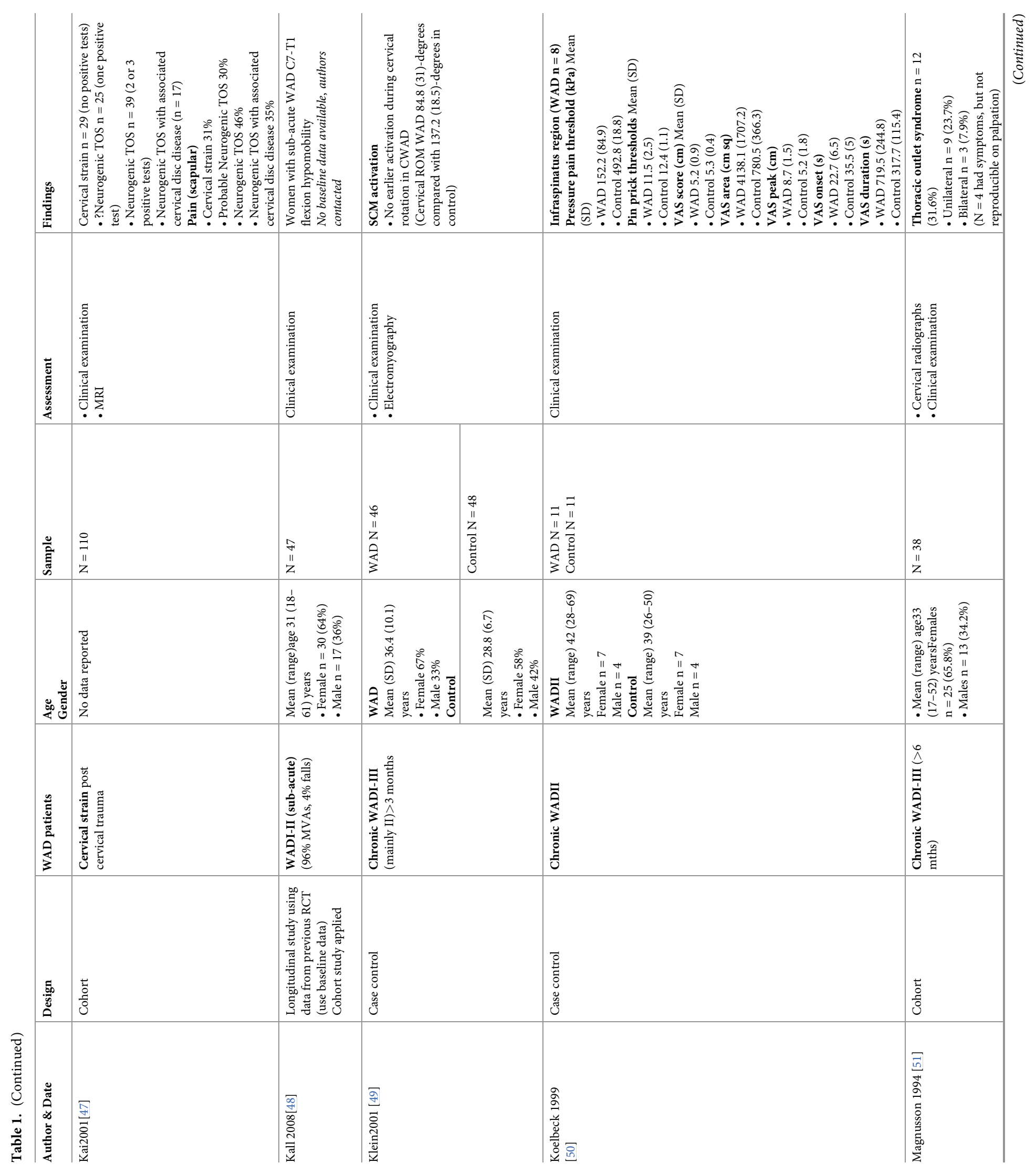




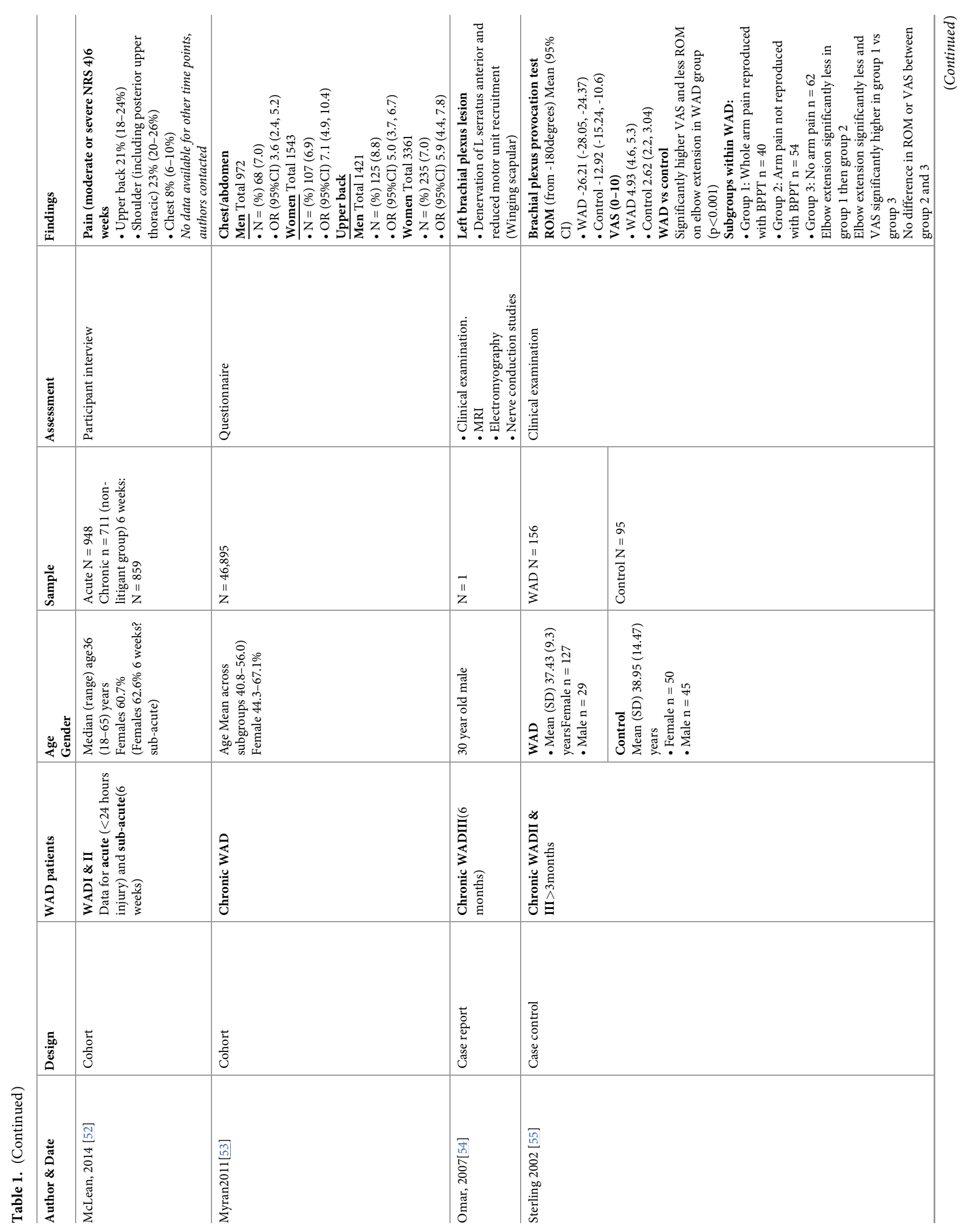




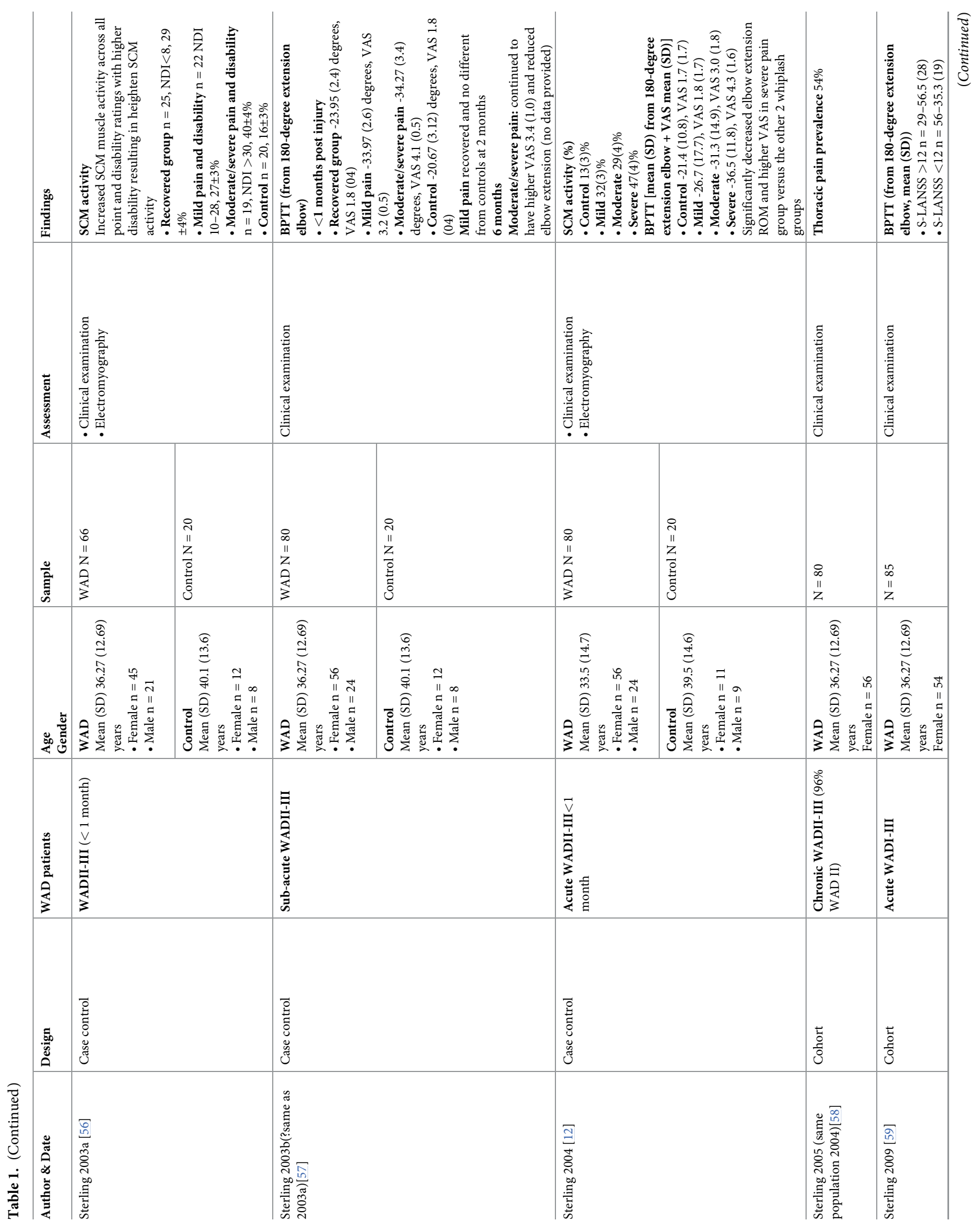


PLUS | ONE

Thoracic dysfunction in WAD

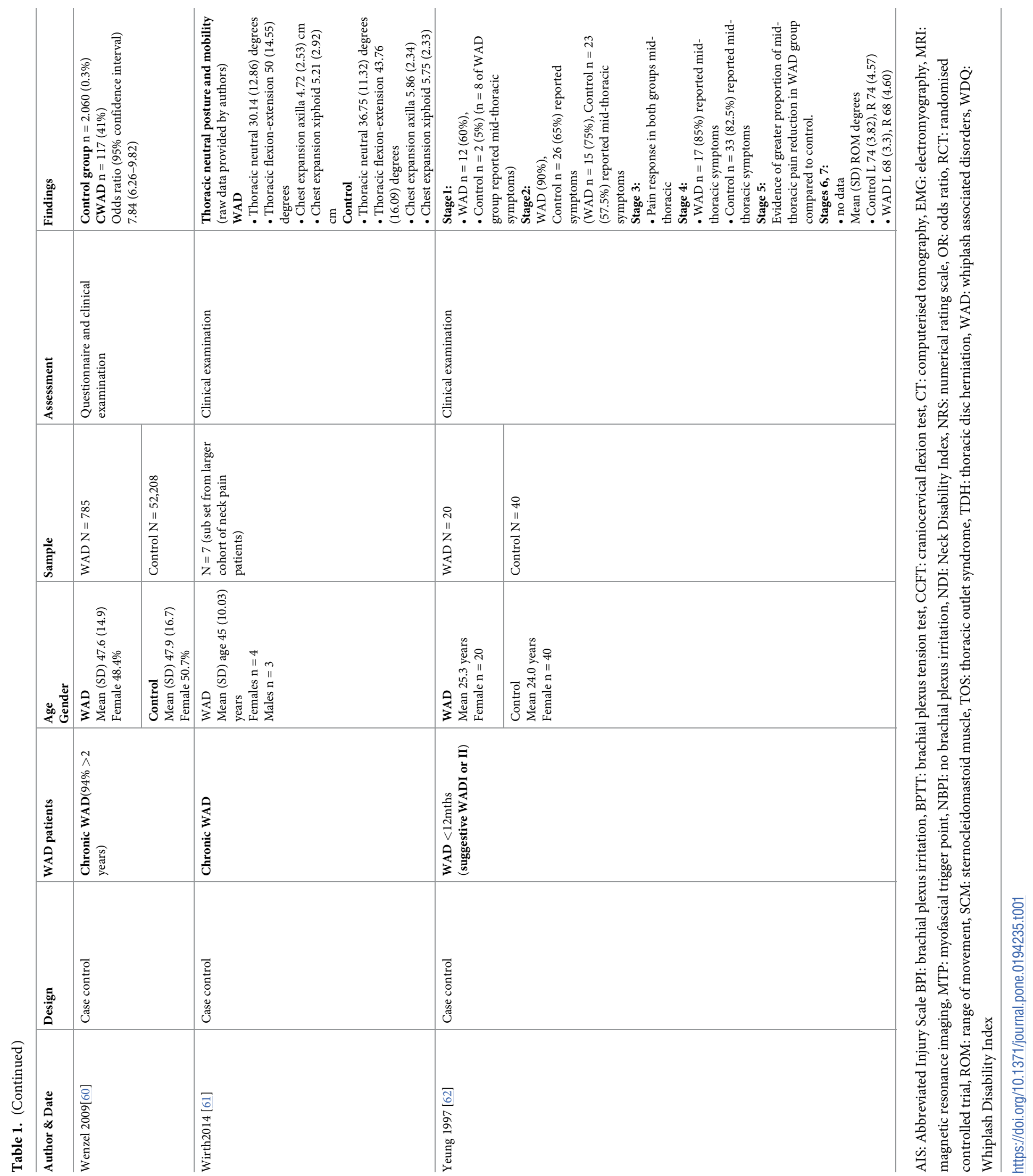

PLOS ONE | https://doi.org/10.1371/journal.pone.0194235 March 23, 2018

$13 / 35$ 
Table 2. Overview of included studies.

\begin{tabular}{|c|c|c|c|c|c|c|c|}
\hline $\begin{array}{c}\text { Author } \\
\mathrm{N}=\mathbf{3 7} \text { papers }\end{array}$ & Measurement and summary outcome & $\begin{array}{l}\text { Sample size } \\
\quad \mathbf{n}=\end{array}$ & $\begin{array}{c}\text { Acute/ } \\
\text { sub } \\
\text { acute }\end{array}$ & Chronic & $\begin{array}{l}\text { WADI/ } \\
\text { II }\end{array}$ & WADII & $\begin{array}{l}\text { WADII/ } \\
\text { III }\end{array}$ \\
\hline & Thoracic pain prevalence & & & & & & \\
\hline Yeung 1997 & $75 \%$ (during slump) & 20 & & $\mathrm{x}$ & $\mathrm{x} ?$ & & \\
\hline Koelbeck 1999 & No difference light touch (reduced PPT, hyperalgesia \& referral) & 11 & & $\mathrm{x}$ & & $\mathrm{x}$ & \\
\hline Bergland 2001 & $15.3 \%$ & 242 & & $\mathrm{x}$ & $\begin{array}{c}\mathrm{x} \\
\text { (minor) }\end{array}$ & & \\
\hline Hartling 2002 & $74 \%$ & 380 & $\mathrm{x}$ & & $\mathrm{x}$ & & $\mathrm{x}$ \\
\hline Bock 2005 & $71 \%$ (allodynia) & 22 & & $\mathrm{x}$ & $\mathrm{x}$ & & \\
\hline Sterling 2005 & $54 \%$ & ***76 & & $\mathrm{x}$ & & & $\mathrm{x}$ \\
\hline Holm 2007 & Acute $52 \%$, chronic $80 \%$ & 56 & $\mathrm{x}$ & $\mathrm{x}$ & & & \\
\hline Wenzel 2009 & $41 \%$ & 785 & & $\mathrm{x}$ & & & \\
\hline Hincapie 2010 & $66-75 \%$ & 6481 & $\mathrm{x}$ & & & & \\
\hline Myran 2011 & $7-8.8 \%$ & 46,895 & & $\mathrm{x}$ & & & \\
\hline Bortsov 2014 & $47 \%$ & $* 948$ & $\mathrm{x}$ & & $\mathrm{x}$ & & \\
\hline Cornips 2014 & Pain associated with herniated disc $4 / 10$ & 10 & & $x$ & & & \\
\hline McLean 2014 & $21 \%$ (upper thoracic/shoulder 23\%) & 711 & $\mathrm{x}$ & & $\mathrm{x}$ & & \\
\hline \multirow[t]{2}{*}{ Johansson 2015} & $43 \%$ & 3711 & $\mathrm{x}$ & $?$ & & & \\
\hline & Chest pain prevalence & & & & & & \\
\hline Holm 2007 & Acute $0 \%$, chronic $6 \%$ & 56 & $\mathrm{x}$ & $\mathrm{x}$ & & & \\
\hline Hincapie $2010^{*}$ & $19 \%$ & 6481 & $\mathrm{x}$ & & & & \\
\hline Myran 2011 & $6.9-7 \%$ & 46,895 & & $\mathrm{x}$ & & & \\
\hline Bortsov $2014^{*}$ & $19 \%$ & 948 & $\mathrm{x}$ & & $\mathrm{x}$ & & \\
\hline \multirow[t]{2}{*}{ McLean $2014^{*}$} & $8 \%$ & 948 & $\mathrm{x}$ & & $\mathrm{x}$ & & \\
\hline & Thoracic posture \& mobility & & & & & & \\
\hline Kall 2008 & Flexion hypomobility & 47 & $\mathrm{x}$ & & $\mathrm{x}$ & & $\mathrm{x}$ \\
\hline Helgadottir 2011a & No change & 23 & & $\mathrm{x}$ & & $\mathrm{x}$ & \\
\hline \multirow[t]{2}{*}{ Wirth 2014} & Reduced kyphosis (chest expansion) & 7 & & $\mathrm{x}$ & & & \\
\hline & Thoracic outlet syndrome prevalence & & & & & & \\
\hline Capistrant 1976 & $86 \%$ & 35 & & $\mathrm{x}$ & & & \\
\hline Capistrant 1986 & $36 \%$ & 111 & & $\mathrm{x} ?$ & & & \\
\hline Magnusson 1994 & $32 \%$ & 38 & & $\mathrm{x}$ & $\mathrm{x}$ & & $\mathrm{x}$ \\
\hline Kai 2001 & $74 \%$ & 110 & & & & & \\
\hline \multirow[t]{2}{*}{ Alexandre 2005} & Positive & 24 & & $\mathrm{x}$ & $\mathrm{x}$ (mild) & & \\
\hline & Brachial Plexus test & & & & & & \\
\hline Ide 2001 & Prevalence $38 \%$ & 119 & $\mathrm{x}$ & $\mathrm{x}$ & & & \\
\hline Sterling 2002 & Positive & 156 & & $\mathrm{x}$ & & & $\mathrm{x}$ \\
\hline Sterling $2003 \mathrm{~b}$ & Positive & ${ }^{* *} 80$ & $\mathrm{x}$ & $\mathrm{x}$ & & & $\mathrm{x}$ \\
\hline Sterling 2004 & Positive & 80 & $\mathrm{x}$ & & & & $\mathrm{x}$ \\
\hline Omar 2007 & Brachial plexus lesion & 1 & & $x$ & & & $x$ \\
\hline Chien 2009 & Positive & 31 & & $\mathrm{x}$ & & $\mathrm{x}$ & \\
\hline Sterling 2009 & Positive & 85 & $\mathrm{x}$ & & & & $\mathrm{x}$ \\
\hline \multirow[t]{2}{*}{ Ferrari 2010} & Positive & 69 & $\mathrm{x}$ & & $\mathrm{x}$ & & \\
\hline & Muscle activation & & & & & & \\
\hline Bodack 1998 & Weak mid and lower trapezius & 1 & $x$ & & & & $x$ \\
\hline Klein 2001 & SCM muscle activation: normal & 46 & & $\mathrm{x}$ & & $\begin{array}{c}\mathrm{x} \\
\text { (mainly) }\end{array}$ & \\
\hline Sterling 2003a & SCM muscle activity heightened & ${ }^{* *} 66$ & $\mathrm{x}$ & & & & $\mathrm{x}$ \\
\hline
\end{tabular}


Table 2. (Continued)

\begin{tabular}{|c|c|c|c|c|c|c|c|}
\hline $\begin{array}{c}\text { Author } \\
\mathrm{N}=\mathbf{3 7} \text { papers }\end{array}$ & Measurement and summary outcome & $\begin{array}{l}\text { Sample size } \\
\quad \mathrm{n}=\end{array}$ & $\begin{array}{c}\text { Acute/ } \\
\text { sub } \\
\text { acute }\end{array}$ & Chronic & $\begin{array}{c}\text { WADI/ } \\
\text { II }\end{array}$ & WADII & $\begin{array}{l}\text { WADII/ } \\
\text { III }\end{array}$ \\
\hline Sterling 2004 & SCM muscle activity heightened & ${ }^{* * *} 80$ & $\mathrm{x}$ & & & & $\mathrm{x}$ \\
\hline \multirow[t]{2}{*}{ Helgadottir 2011b } & $\begin{array}{l}\text { Serratus anterior activation: delayed onset and reduced duration of } \\
\text { activation. No change for lower and mid trapezius }\end{array}$ & 27 & & $\mathrm{x}$ & & $\mathrm{x}$ & \\
\hline & Muscle pain and trigger points & & & & & & \\
\hline Bismil 2005 & Mid/Low trapezius enthesopathy (myofascial pain $+\mathrm{TP}$ ) $48 \%$ & 25 & & $\mathrm{x}$ & & $\mathrm{x}$ & \\
\hline Ettlin 2008 & SCM and Scaleneus medius MTP prevalence $24 \%, 30 \%$ & 47 & & $\mathrm{x}$ & & & \\
\hline $\begin{array}{l}\text { Fernandez-de-las- } \\
\text { Perez } 2012\end{array}$ & SCM and Scaleneus medius MTP & 20 & $\mathrm{x}$ & & & $\mathrm{x}$ & \\
\hline Castaldo 2014 & SCM Latent and active TP & 49 & & $\mathrm{x}$ & $\mathrm{x}$ (mild) & & $\mathrm{x}$ \\
\hline \multicolumn{8}{|l|}{ Note } \\
\hline \multicolumn{8}{|l|}{${ }^{*}$ Same population } \\
\hline \multicolumn{8}{|l|}{ ** same population } \\
\hline \multicolumn{8}{|l|}{ *** same population } \\
\hline \multicolumn{8}{|c|}{ SCM: sternocleidomastoid muscle } \\
\hline
\end{tabular}

https://doi.org/10.1371/journal.pone.0194235.t002

specifically associated with central sensitisation (allodynia) where pain prevalence was $71 \%$ in individuals with CWADI/II [33].

Chest pain. Prevalence of chest pain in acute/sub-acute was reported to range $0.0-19 \%$ $[11,14,17,52]$, although when considered as part of a widespread pain presentation ranged 9-19\% [11, 14]. In one study where they only considered individuals with numerical rating scale (NRS 0-10) 4+ chest pain prevalence was lower at 8\% [52]. In CWAD prevalence rates for chest pain ranged $6-22 \%[17,53]$ although this reflects a, broad timescale with one study reporting results 6 month post injury [17] to one exploring prevalence at any time point following injury [53]. Drawing definitive conclusions on prevalence of chest pain is difficult given variation in approaches used to record pain (pain drawings, VAS, etc.), time points post injury, sample heterogeneity and the overall methodological low quality of research. This may also be a related to the focus of this review, being to those with mild to moderate presentations of WAD where studies including WADIV or fractures were excluded.

Thoracic posture. Evidence of thoracic postural dysfunction is inconclusive given conflicting findings from a small number of studies of low methodological quality evidence where postural assessment was not a primary focus $[44,61]$. Future studies should consider the use of a gold standard measure for postural evaluation [63].

Thoracic mobility and chest mobility. There is a notable gap in the evidence exploring thoracic and chest mobility in WAD, with just two studies $(n=54)$ of very low quality suggesting a trend for reduced chest mobility $(\mathrm{p}>0.05)$ and flexion hypomobility at the cervico-thoracic junction in women. In terms of thoracic mobility, evidence from one study $(n=7)$ [61] suggests a slight increase in thoracic flexion-extension, although with such a small sample and lack of inclusion of a valid approach to quantify thoracic mobility, meaningful conclusions cannot drawn.

Thoracic outlet syndrome in CWAD. Five studies found, a prevalence of 31-74+\% [31, $35,36,47,51]$ of thoracic outlet syndrome in CWAD. Although this suggests a relatively high prevalence, the methodological quality of included studies was generally poor $(<4 / 9)$, resulting in a very low rating of quality according to GRADE.

Brachial plexus. Acute/sub-acute WAD 


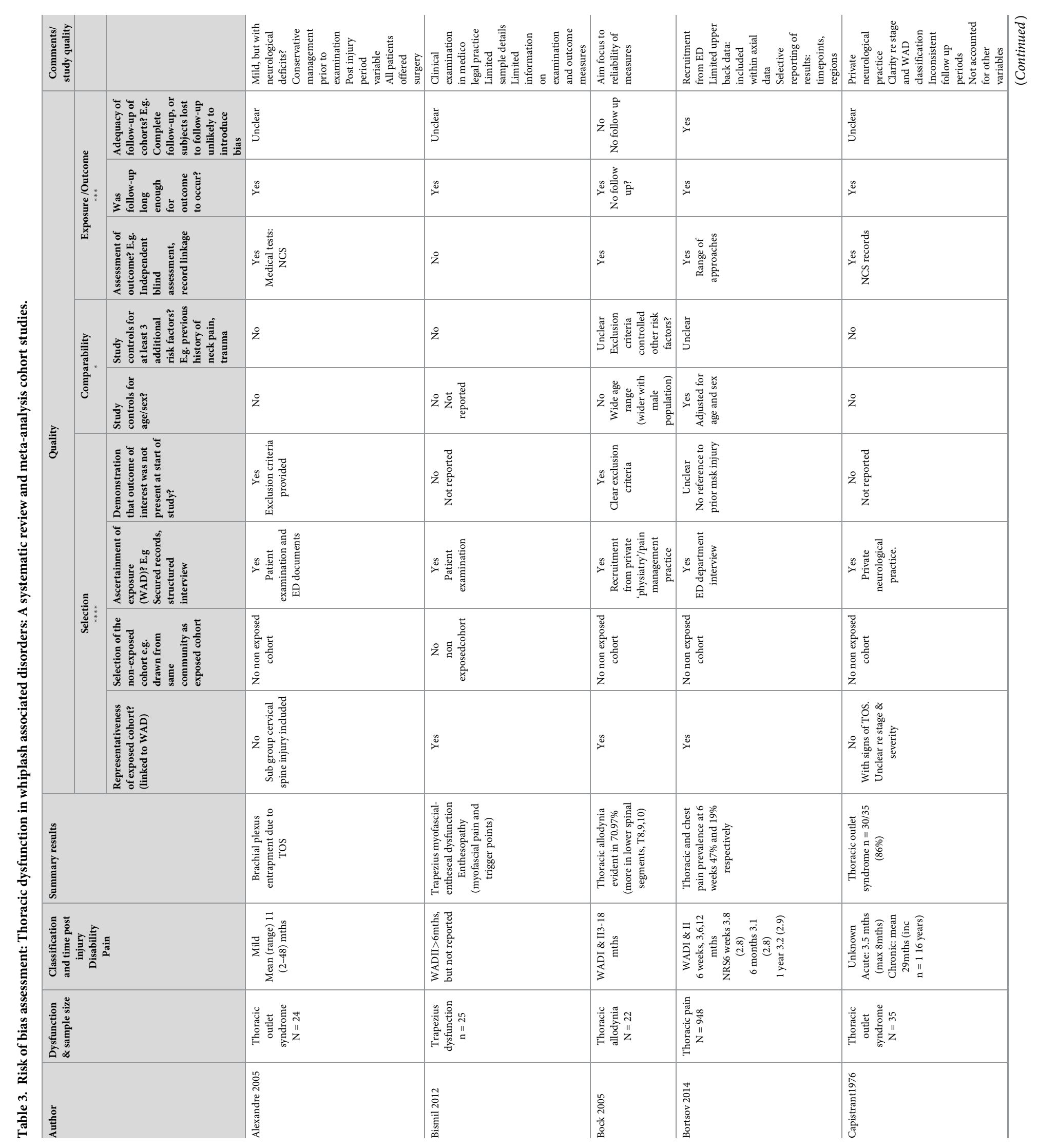




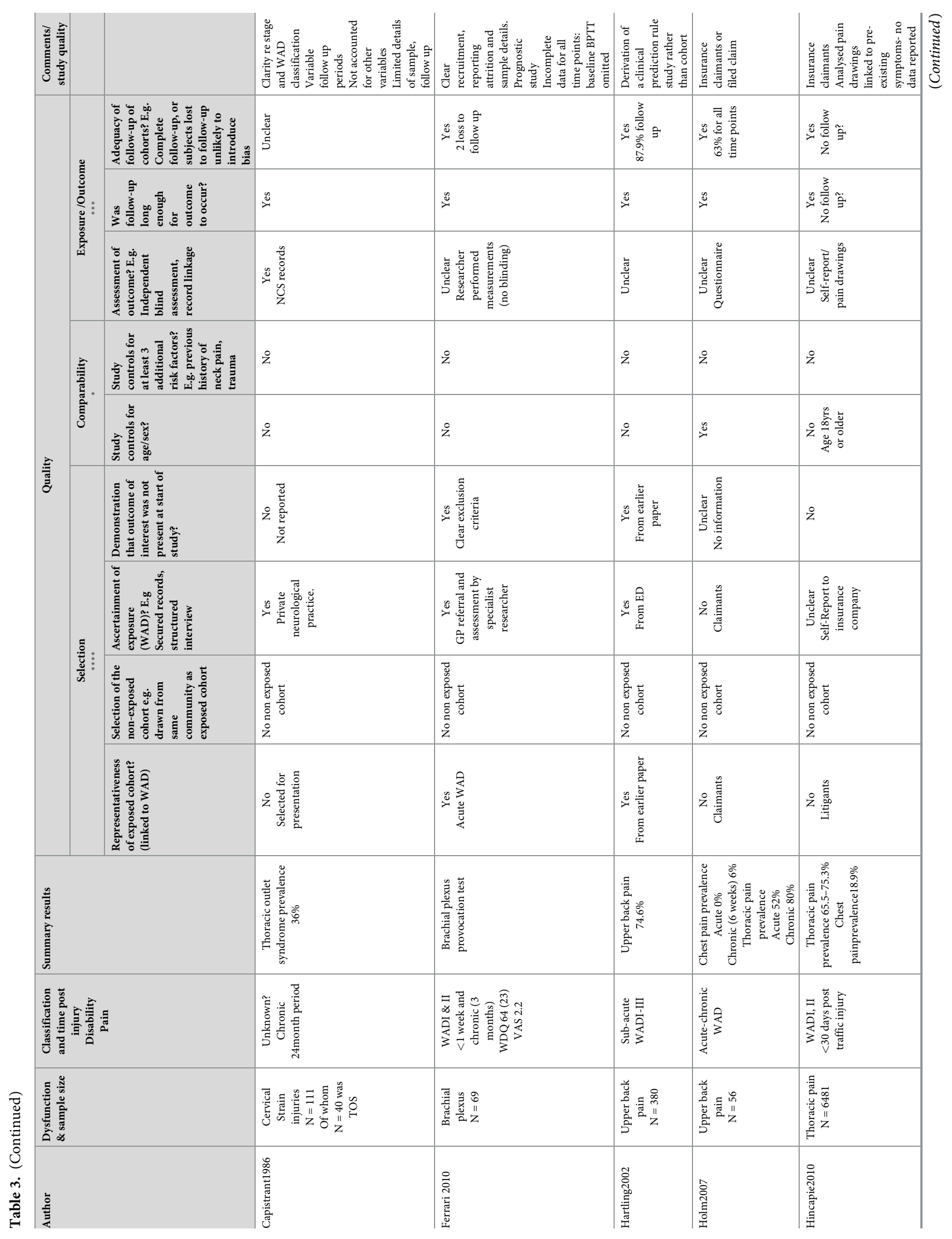




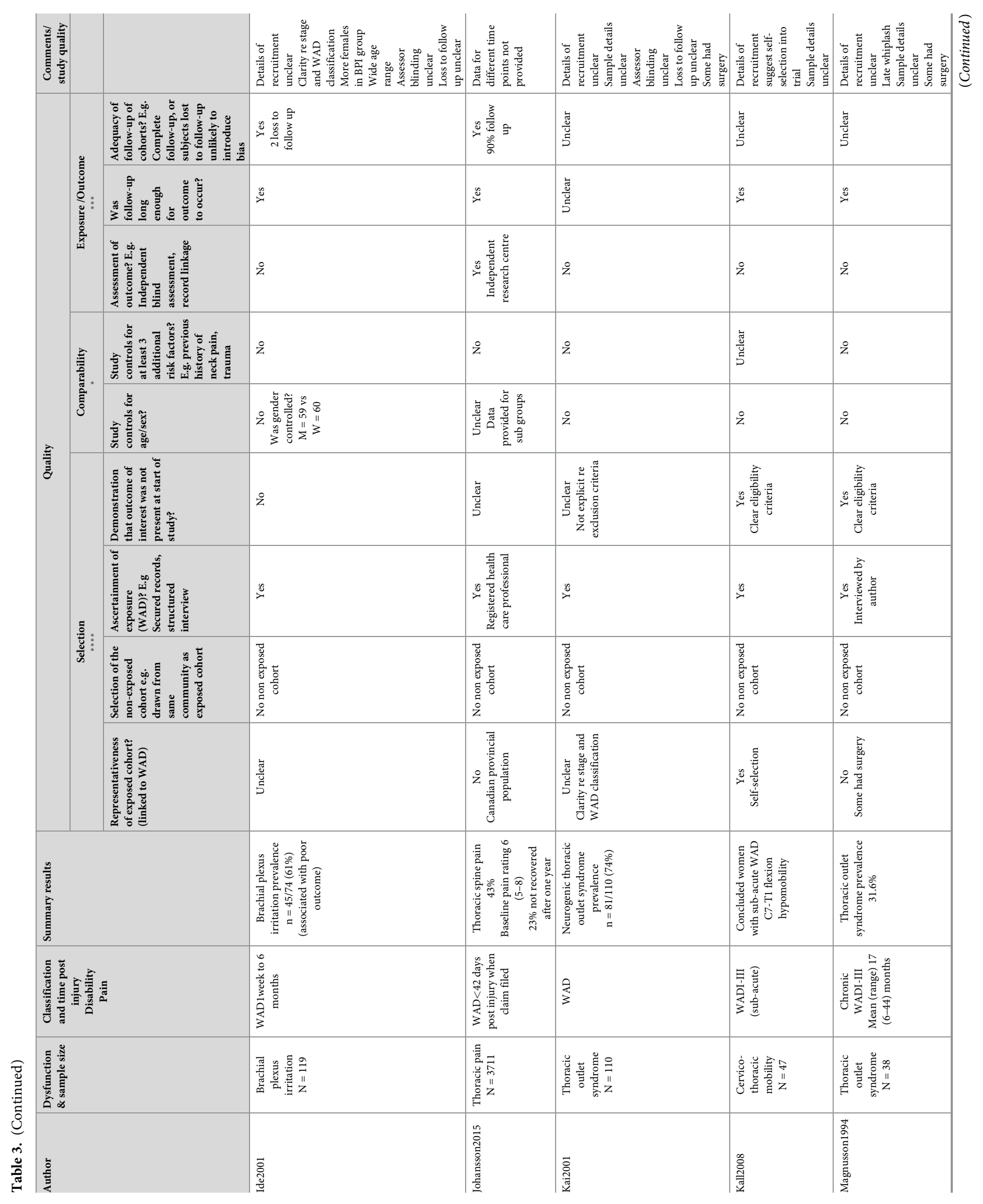




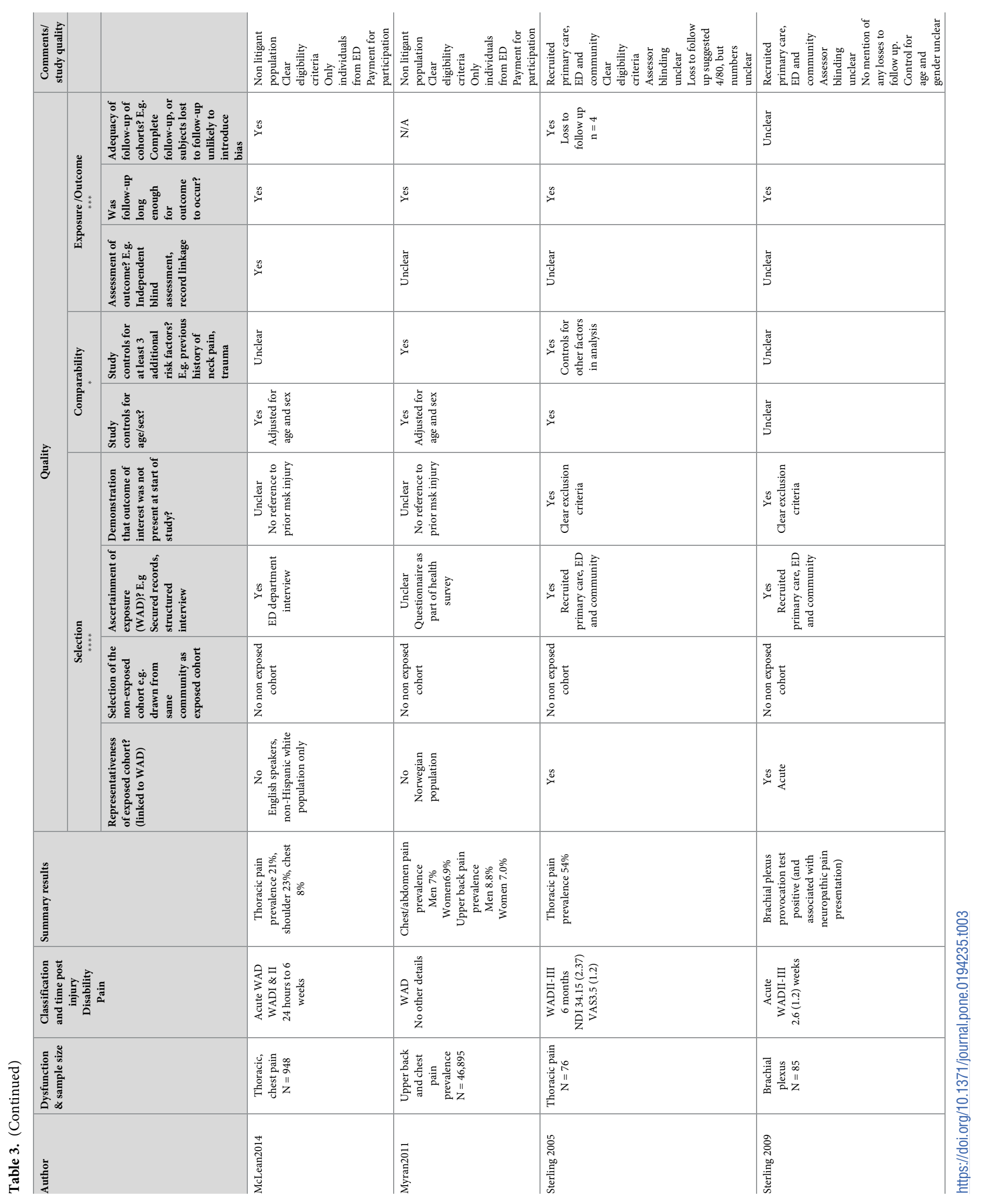




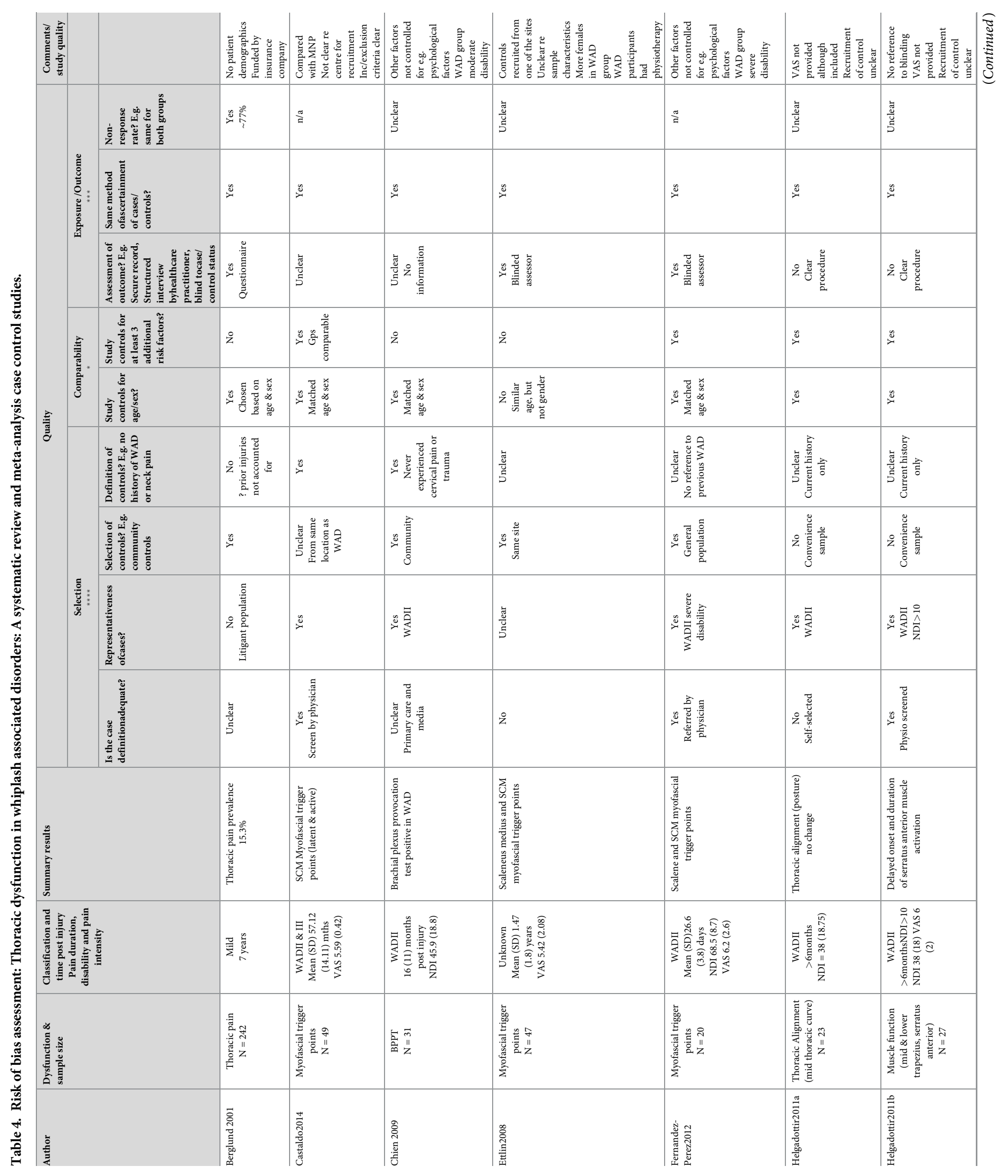




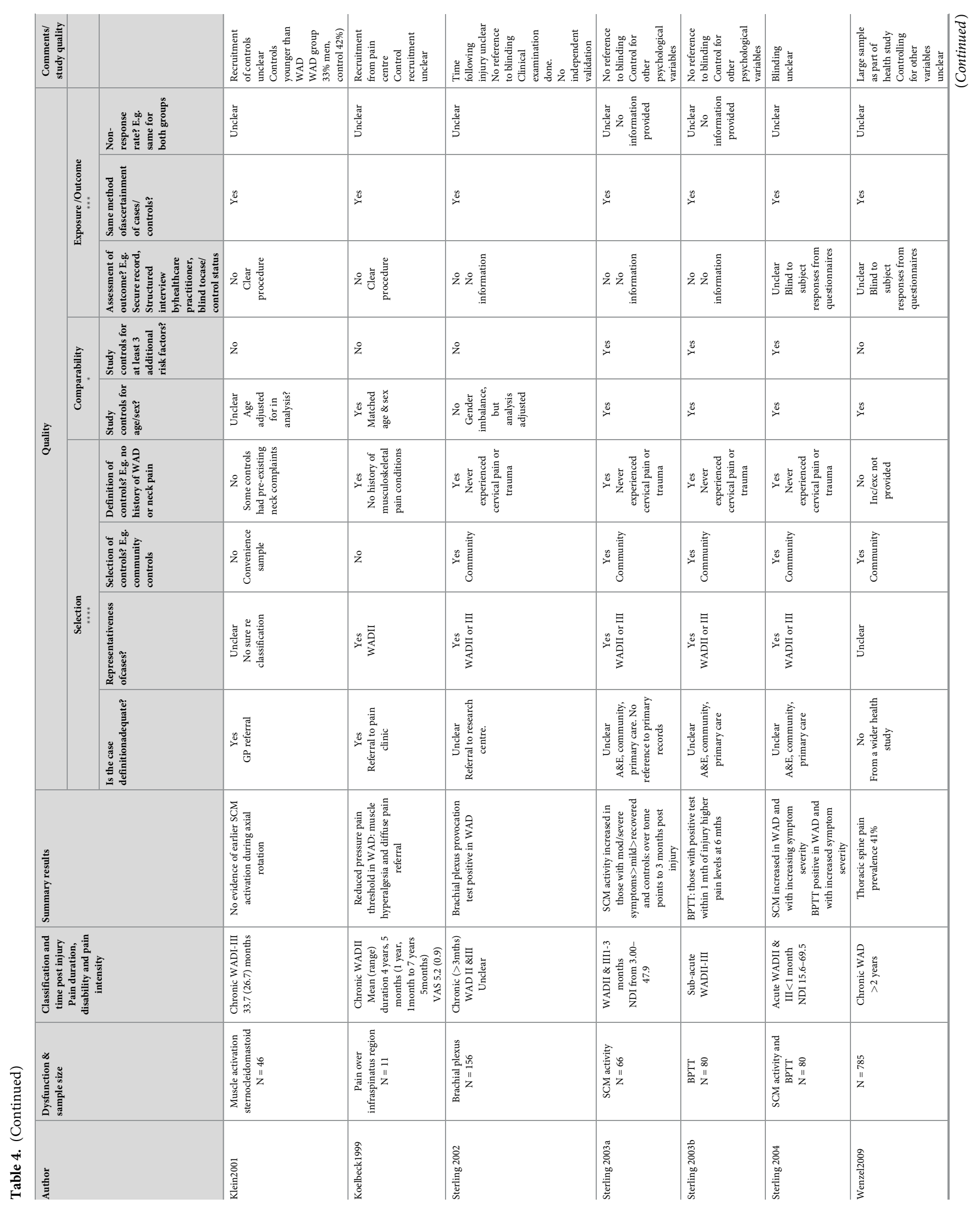




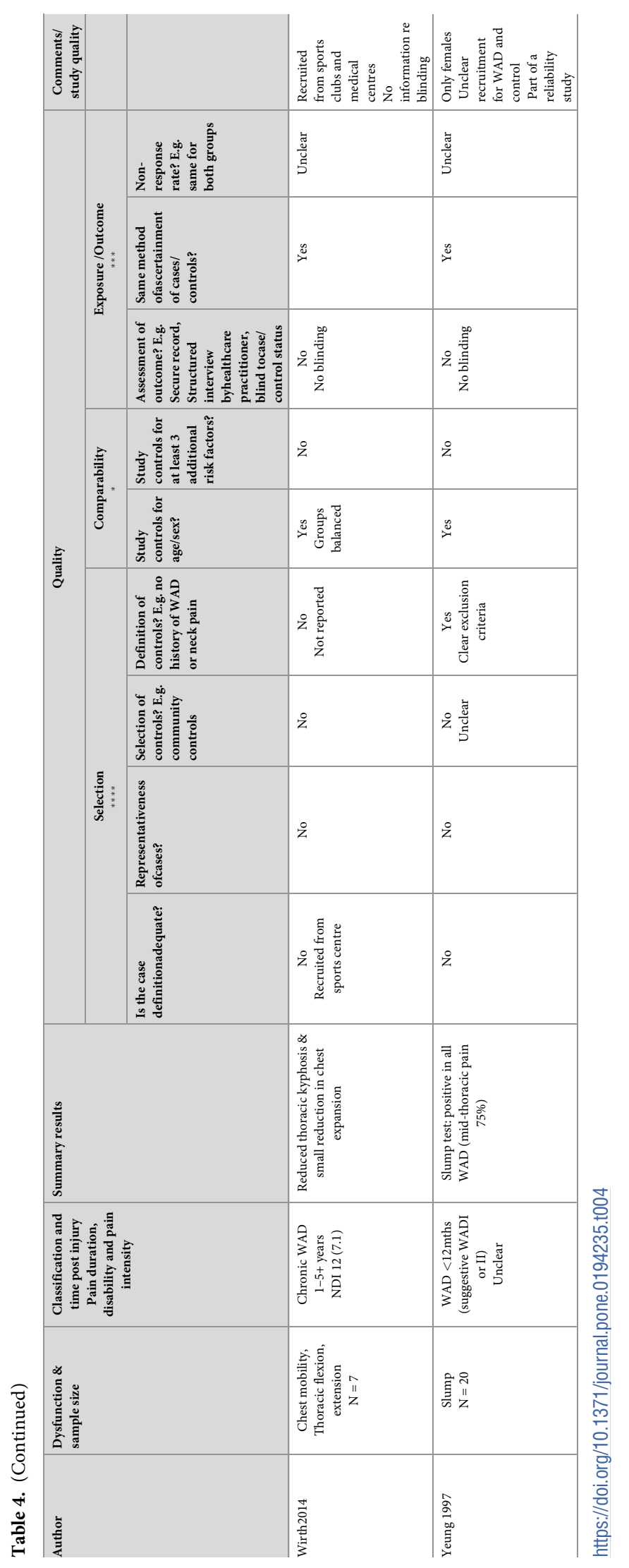


Table 5. Pain.

\begin{tabular}{|c|c|c|c|c|}
\hline Outcome & Studies & $\begin{array}{l}\text { Findings Prevalence \% } \\
\text { (95\% confidence interval) } \\
\text { unless stated }\end{array}$ & Studies & Overall GRADE \\
\hline \multirow{7}{*}{$\begin{array}{l}\text { Thoracic spine } \\
\text { pain in acute/sub- } \\
\text { acute WAD } \\
\mathrm{N}=11,577\end{array}$} & Bodack 1998 & -Positive & \multirow{7}{*}{$\begin{array}{l}6 \text { cohort, } \\
1 \text { single case } \\
\text { study }\end{array}$} & \multirow{7}{*}{$\begin{array}{l}\text { Very low-due to risk of } \\
\text { bias, directness and } \\
\text { precision }\end{array}$} \\
\hline & Hartling 2002 & -Upper back pain $74.6 \%$ & & \\
\hline & Holm 2007 & $-52 \%$ & & \\
\hline & Hincapie 2010 & $\begin{array}{l}\text {-Mid back } 65.5(64.4- \\
66.7) \\
\text {-Upper thoracic } 75.3 \\
(74.3-76.4)\end{array}$ & & \\
\hline & a Bortsov 2014 (Mild) & $\begin{array}{l}\text {-Mid back } 47 \%(18 \% \\
\text { widespread, } 29 \% \\
\text { localised) }\end{array}$ & & \\
\hline & $\begin{array}{l}{ }^{\text {a }} \text { McLean } 2014 \\
\text { (WADI/II) (moderate } \\
\text { or severe pain NRS4) }\end{array}$ & $\begin{array}{l}\text {-Mid back 21\% (range } \\
18-24 \% \text { ) } \\
\text {-Upper back 23\% (range } \\
20-26 \% \text { ) }\end{array}$ & & \\
\hline & Johansson 2015 & $-43 \%$ & & \\
\hline \multirow{9}{*}{$\begin{array}{l}\text { Thoracic spine } \\
\text { pain in chronic } \\
\text { WAD } \\
N=48,117\end{array}$} & $\begin{array}{l}\text { Yeung 1997(?WADI/ } \\
\text { II) }\end{array}$ & -Slump & \multirow{9}{*}{$\begin{array}{l}5 \text { cohort, } 3 \\
\text { case control, } 1 \\
\text { case series }\end{array}$} & \multirow{9}{*}{$\begin{array}{l}\text { Very low-due to risk of } \\
\text { bias, precision, } \\
\text { consistency and } \\
\text { directness }\end{array}$} \\
\hline & \begin{tabular}{|l|}
$\begin{array}{l}\text { Koelbeck } 1999 \\
\text { (WADII) }\end{array}$ \\
\end{tabular} & -No different controls & & \\
\hline & \begin{tabular}{|l} 
Bergland 2001 \\
(Minor)
\end{tabular} & $-15.3 \%(10.4-21.5)$ & & \\
\hline & Bock 2005 (WADI/II) & $-71 \%$ (allodynia) & & \\
\hline & \begin{tabular}{|l} 
Sterling 2005 \\
(WADII/III)
\end{tabular} & $-54 \%$ & & \\
\hline & Holm 2007 & $\begin{array}{l}-4 \text { months } 86 \% \text {, } \\
-8 \text { months } 94 \% \text {, } \\
-12 \text { months } 87 \% \\
\end{array}$ & & \\
\hline & Wenzel 2009 & $-41 \%$ & & \\
\hline & Myran 2011 & $\begin{array}{l}\text {-men } 8.8 \% \\
\text {-women } 7 \% \\
\end{array}$ & & \\
\hline & Cornips 2014 & $\begin{array}{l}10 \text { previously } \\
\text { asymptomatic individuals } \\
\text { significant pain }\end{array}$ & & \\
\hline \multirow{4}{*}{$\begin{array}{l}\text { Thoracic spine } \\
\text { pain in chronic } \\
\text { WAD I/II } \\
\mathrm{N}=655\end{array}$} & \begin{tabular}{|l}
$\begin{array}{l}\text { Koelbeck } 1999 \\
\text { (WADII) }\end{array}$ \\
\end{tabular} & -No different controls & \multirow[t]{4}{*}{$\begin{array}{l}3 \text { cohort, } 1 \\
\text { case control }\end{array}$} & \multirow{4}{*}{$\begin{array}{l}\text { Very low-due to risk of } \\
\text { bias, precision, } \\
\text { consistency and } \\
\text { directness }\end{array}$} \\
\hline & $\begin{array}{l}\text { Bergland } 2001 \\
\text { (Minor) }\end{array}$ & $-15.3 \%(10.4-21.5)$ & & \\
\hline & Hartling 2002 & -Upper back pain $74.6 \%$ & & \\
\hline & Bock 2005 (WADI/II) & $-71 \%$ (allodynia) & & \\
\hline $\begin{array}{l}\text { Thoracic spine } \\
\text { pain in chronic } \\
\text { WADII/III } \\
\mathrm{N}=76\end{array}$ & $\begin{array}{l}\text { Sterling } 2005 \\
\text { (WADII/III) }\end{array}$ & $-54 \%$ & 1 cohort & Low?? \\
\hline \multirow{4}{*}{$\begin{array}{l}\text { Chest pain in } \\
\text { acute/sub-acute } \\
\text { WAD } \\
N=7485\end{array}$} & Holm 2007 & $-0 \%$ & \multirow[t]{4}{*}{4 Cohorts } & \multirow{4}{*}{$\begin{array}{l}\text { Very low-due to risk of } \\
\text { bias, precision, } \\
\text { consistency and } \\
\text { directness }\end{array}$} \\
\hline & Hincapie 2010 & $\begin{array}{l}\text {-localised } 0.05(0.00-0.10) \\
\text {-widespread } 18.9(17.9- \\
19.8)\end{array}$ & & \\
\hline & aBortsov 2014 (mild) & $\begin{array}{l}-19 \%(10 \% \text { localised } \\
-9 \% \text { widespread })\end{array}$ & & \\
\hline & $\begin{array}{l}\text { aMcLean } 2014 \\
\text { (WADI/II) }\end{array}$ & $\begin{array}{l}-8 \%(6-10 \%)(\text { moderate } \\
\text { or severe pain) }\end{array}$ & & \\
\hline
\end{tabular}


Table 5. (Continued)

\begin{tabular}{|c|c|c|c|c|}
\hline Outcome & Studies & $\begin{array}{l}\text { Findings Prevalence \% } \\
(95 \% \text { confidence interval) } \\
\text { unless stated }\end{array}$ & Studies & Overall GRADE \\
\hline \multirow[t]{2}{*}{$\begin{array}{l}\text { Chest pain in } \\
\text { chronic WAD } \\
\mathrm{N}=46,951\end{array}$} & Holm 2007 & $\begin{array}{l}\text {-6-weeks } 6 \% \\
-4 \text {-months } 4 \% \\
8 \text {-months } 12 \% \\
\text {-12-months } 22 \%\end{array}$ & \multirow[t]{2}{*}{2 cohorts } & \multirow[t]{2}{*}{$\begin{array}{l}\text { Very low-due to risk o } \\
\text { bias, precision, } \\
\text { directness }\end{array}$} \\
\hline & Myran 2011 & -men $7 \%$, women $6.9 \%$ & & \\
\hline
\end{tabular}

https://doi.org/10.1371/journal.pone.0194235.t005

Notwithstanding that evidence is drawn principally from one research group, there is evidence of thoracic dysfunction in relation to the brachial plexus provocation test (BPPT) in WADI-III. From the research by Sterling et al [12, 57, 59], there is evidence of a negative association between self-report symptom severity (VAS) and range of elbow extension during the BPPT in chronic WAD. Moreover, the extent of this dysfunction is in turn related to the degree of pain and disability, with those participants presenting with higher levels of selfreported pain and disability (NDI) having greater levels of dysfunction during BPPT.

\section{Chronic WAD}

For participants with CWADII and/or III, evidence indicates dysfunction detected during the BPPT, with pain levels and restriction in elbow extension almost double those found in asymptomatic controls $[38,55]$. Furthermore, those with higher levels of self-reported pain and disability (NDI $>30$ ) continued to have evidence of dysfunction 6 months after the injury which was not seen in the mild group who were no different to the recovered group at 2 months [57]. Although quality of individual studies varied (4-6/9), the overall body of evidence for dysfunction of the brachial plexus remains very low overall, primarily due to risk of bias of the included studies.

Muscle activation. There are limited and very variable findings of thoracic muscle dysfunction (activation) in WAD with studies investigating a relatively small number of muscles: sternocleidomastoid [12, 49, 56]; serratus anterior [45]; middle and lower fibres of trapezius $[45,54]$. Although it is difficult to derive meaningful conclusions with respect to serratus anterior and trapezius, there is evidence supporting changes in sternocleidomastoid muscle activation with heightened levels of activation during a task of cranio-cervical flexion; this increase in sternocleidomastoid activity however, was not seen during neck rotation [49]. There appears to be a positive relationship between sternocleidomastoid activation and higher levels of pain severity, with participants with CWADII/III and moderate to severe levels of disability demonstrating increased levels of sternocleidomastoid activation of between $27-47 \%[12,56]$.

Table 6. Posture and mobility.

\begin{tabular}{|c|c|c|c|c|}
\hline Outcome & Studies & Findings & Grade & Overall GRADE \\
\hline \multirow{2}{*}{$\begin{array}{l}\text { Thoracic posture in } \\
\text { chronic WAD } \\
\mathrm{N}=30\end{array}$} & $\begin{array}{l}\text { Helgadottir } \\
\text { 2011a (WADII) }\end{array}$ & -No change & \multirow[t]{2}{*}{2 Case control } & \multirow{2}{*}{$\begin{array}{l}\text { Very low: due to risk of bias, } \\
\text { precision, consistency and } \\
\text { directness }\end{array}$} \\
\hline & Wirth 2014 & -Reduced kyphosis & & \\
\hline \multirow{2}{*}{$\begin{array}{l}\text { Thoracic and chest } \\
\text { mobility in chronic } \\
\text { WAD } \\
N=54\end{array}$} & Kall 2008 & $\begin{array}{l}\text {-Flexion } \\
\text { hypomobility in } \\
\text { women }\end{array}$ & \multirow[t]{2}{*}{$\begin{array}{l}1 \text { cohort } \\
\text { study, } 1 \text { case } \\
\text { control }\end{array}$} & \multirow[t]{2}{*}{$\begin{array}{l}\text { Very low: due to risk of bias, } \\
\text { precision, consistency and } \\
\text { directness }\end{array}$} \\
\hline & Wirth 2014 & $\begin{array}{l}\text {-Reduced chest } \\
\text { mobility } \\
\text {-Slight increase in } \\
\text { thoracic mobility }\end{array}$ & & \\
\hline
\end{tabular}

https://doi.org/10.1371/journal.pone.0194235.t006 
Table 7. Thoracic outlet syndrome and brachial plexus.

\begin{tabular}{|c|c|c|c|c|}
\hline Outcome & Studies & $\begin{array}{l}\text { Findings [TOS: Prevalence \% (95\% confidence interval); } \\
\text { Brachial plexus provocation test (BPPT) unless stated] }\end{array}$ & Grade & Overall GRADE \\
\hline \multirow{5}{*}{$\begin{array}{l}\text { Thoracic outlet } \\
\text { syndrome in chronic } \\
\text { WAD } \\
\mathrm{N}=318\end{array}$} & Capistrant 1976 & $-86 \%$ & \multirow[t]{5}{*}{$\begin{array}{l}5 \text { Cohort } \\
\text { studies }\end{array}$} & \multirow{5}{*}{$\begin{array}{l}\text { Very low: due to risk of bias, } \\
\text { directness, precision, reporting } \\
\text { bias }\end{array}$} \\
\hline & Capistrant 1986 & $-36 \%$ & & \\
\hline & Magnusson 1994 & $-31.6 \%$ & & \\
\hline & Kai 2001 & $74 \%$ & & \\
\hline & Alexandre 2005 & -Positive secondary to BPI & & \\
\hline \multirow{8}{*}{$\begin{array}{l}\text { Brachial Plexus in all } \\
\text { WAD } \\
(\mathbf{n}=620)\end{array}$} & Ide 2001 & BPI 38\% & \multirow{8}{*}{$\begin{array}{l}4 \text { case } \\
\text { control,3 } \\
\text { cohort }\end{array}$} & \multirow{8}{*}{$\begin{array}{l}\text { Very low: due to risk or bias, } \\
\text { directness and precision }\end{array}$} \\
\hline & $\begin{array}{l}\text { Sterling } 2002 \text { (WADII/ } \\
\text { III) } \\
\text { Chronic }\end{array}$ & $\begin{array}{l}\text {-WAD -26.21 (95\%CI -28.05, -24.37), VAS } 4.93(4.6,5.3) \\
\text {-Control -12.92 (95\%CI -15.24, -10.6), VAS } 2.62(2.2, \\
3.04)\end{array}$ & & \\
\hline & $\begin{array}{l}\text { Sterling } 2003 \mathrm{~b} \text { (WADII/ } \\
\text { III) } \\
\text { Acute/sub-acute }\end{array}$ & $\begin{array}{l}<\mathbf{1} \text { months post injury [mean (SD) } \\
\text { - Recovered group -23.95 (2.4) degrees, VAS } 1.8(04) \\
\text { - Mild pain -33.97 (2.6) degrees, VAS 3.2 (0.5) } \\
\text { - Moderate/severe pain -34.27 (3.4) degrees, VAS } 4.1(0.5) \\
\text { - Control -20.67 (3.12) degrees, VAS } 1.8(04) \\
\text { Mild pain recovered and no different from controls at } 2 \\
\text { months }\end{array}$ & & \\
\hline & $\begin{array}{l}\text { Sterling 2003b (WADII/ } \\
\text { III) } \\
\text { Chronic }\end{array}$ & $\begin{array}{l}6 \text { months } \\
\text { Moderate/severe pain: continued to have higher VAS } 3.4 \\
\text { (1.0) and reduced elbow extension (no data provided) }\end{array}$ & & \\
\hline & $\begin{array}{l}\text { Sterling } 2004 \text { (WADII/ } \\
\text { III) } \\
\text { Acute/sub-acute }\end{array}$ & $\begin{array}{l}\text { Mean (SD) degrees/VAS } \\
\text { - Mild symptoms -26.7 (17.7), VAS 1.8 (1.7) } \\
\text { - Moderate symptoms -31.3 (14.9), VAS 3.0 (1.8) } \\
\text { - Severe symptoms -36.5 (11.8), VAS } 4.3(1.6) \\
\text { - Control -21.4 (10.8), VAS 1.7 (1.7) }\end{array}$ & & \\
\hline & $\begin{array}{l}\text { Sterling } 2009 \text { (WADI-III) } \\
\text { Acute }\end{array}$ & $\begin{array}{l}\text { Mean (SD)degrees } \\
\text { - S-LANSS >12-56.5 (28) } \\
\text { - S-LANSS <12-35.3 (19) }\end{array}$ & & \\
\hline & Chien 2009 (WADII) & $\begin{array}{l}\text { WAD -22.3 (27.4) degrees VAS } 2.4(2.3) \\
\text { Control -11 (5.9) VAS } 0.7(1.1)\end{array}$ & & \\
\hline & Ferrari, 2010 (WADI/II & $\begin{array}{l}3 \text { months: } \\
\text { - VAS: mean (SD)2.2 (1.2) } \\
\text { - Elbow extension: } 41.5(23) \text { degrees }\end{array}$ & & \\
\hline \multirow{5}{*}{$\begin{array}{l}\text { Brachial Plexus In } \\
\text { acute/sub-acute WAD } \\
(\mathrm{n}=419)\end{array}$} & Ide 2001 & BPI 38\% & \multirow{5}{*}{$\begin{array}{l}2 \text { case } \\
\text { control,4 } \\
\text { cohort }\end{array}$} & \multirow{5}{*}{$\begin{array}{l}\text { Very low: due to risk or bias, } \\
\text { directness, precision }\end{array}$} \\
\hline & $\begin{array}{l}\text { Sterling 2003b (WADII/ } \\
\text { III) }\end{array}$ & $\begin{array}{l}<\mathbf{1} \text { months post injury [mean (SD) } \\
\text { - Recovered group (NDI<8) -23.95 (2.4) degrees, VAS } 1.8 \\
(04) \\
\text { - Mild pain (NDI 10-28) -33.97 (2.6) degrees, VAS } 3.2 \\
(0.5) \\
\text { - Moderate/severe (NDI >30) -34.27 (3.4) degrees, VAS } \\
\text { 4.1 (0.5) } \\
\text { - Control -20.67 (3.12) degrees, VAS } 1.8(04)\end{array}$ & & \\
\hline & $\begin{array}{l}\text { Sterling } 2004 \text { (WADII/ } \\
\text { III) }\end{array}$ & $\begin{array}{l}\text { Mean (SD)degrees/VAS (pain \& disability) } \\
\text { - Mild (NDI 15.6) -26.7 (17.7), VAS 1.8 (1.7) } \\
\text { - Moderate (NDI 39.5) -31.3 (14.9), VAS 3.0 (1.8) } \\
\text { - Severe (NDI 69.5)-36.5 (11.8), VAS 4.3 (1.6) } \\
\text { - Control -21.4 (10.8), VAS 1.7 (1.7) }\end{array}$ & & \\
\hline & Sterling 2009 (WADI-III) & $\begin{array}{l}\text { Mean (SD)degrees } \\
\text { - S-LANSS >12-56.5 (28) } \\
\text { - S-LANSS < 12-35.3 (19) }\end{array}$ & & \\
\hline & Ferrari, 2010 (WADI/II) & $\begin{array}{l}3 \text { months: } \\
\text { - VAS: mean (SD)2.2 (1.2) } \\
\text { - Elbow extension: } 41.5(23) \text { degrees }\end{array}$ & & \\
\hline
\end{tabular}


Table 7. (Continued)

\begin{tabular}{|c|c|c|c|c|}
\hline Outcome & Studies & $\begin{array}{l}\text { Findings [TOS: Prevalence \% (95\% confidence interval); } \\
\text { Brachial plexus provocation test (BPPT) unless stated] }\end{array}$ & Grade & Overall GRADE \\
\hline \multirow[t]{5}{*}{$\begin{array}{l}\text { Brachial Plexus In } \\
\text { chronic WAD } \\
(\mathrm{n}=432)\end{array}$} & $\begin{array}{l}\text { Sterling } 2002 \text { (WADII/ } \\
\text { III) }\end{array}$ & $\begin{array}{l}\text {-WAD -26.21 (95\%CI -28.05, -24.37), VAS } 4.93(4.6,5.3) \\
\text {-Control -12.92 (95\%CI -15.24, -10.6), VAS } 2.62(2.2, \\
\text { 3.04) } \\
\text {-Mild (NDI 10-28): recovered and no different from } \\
\text { controls at } 2 \text { months }\end{array}$ & \multirow[t]{5}{*}{$\begin{array}{l}4 \text { case control, } \\
2 \text { cohort }\end{array}$} & \multirow[t]{5}{*}{$\begin{array}{l}\text { Very low: due to risk or bias, } \\
\text { directness, precision }\end{array}$} \\
\hline & $\begin{array}{l}\text { Sterling 2003b (WADII/ } \\
\text { III) }\end{array}$ & $\begin{array}{l}6 \text { months } \\
\text { Moderate/severe (NDI }>30) \text { : continued to have higher } \\
\text { VAS } 3.4(1.0) \text { and reduced elbow extension (no data } \\
\text { provided) }\end{array}$ & & \\
\hline & $\begin{array}{l}\text { Sterling } 2004 \text { (WADII/ } \\
\text { III) }\end{array}$ & $\begin{array}{l}\text { Mean (SD)degrees/VAS (pain \& disability) } \\
\text { - Mild (NDI 15.6) -26.7 (17.7), VAS 1.8 (1.7) } \\
\text { - Moderate (NDI 39.5) -31.3 (14.9), VAS 3.0 (1.8) } \\
\text { - Severe (NDI 69.5)-36.5 (11.8), VAS 4.3 (1.6) } \\
\text { - Control -21.4 (10.8), VAS 1.7 (1.7) }\end{array}$ & & \\
\hline & Chien 2009 (WADII) & $\begin{array}{l}\text { WAD -22.3 (27.4) degrees VAS } 2.4(2.3) \\
\text { Control -11 (5.9) VAS } 0.7(1.1)\end{array}$ & & \\
\hline & Sterling 2009 (WADI-III) & $\begin{array}{l}\text { Mean (SD) degrees } \\
\text { - S-LANSS }>12-56.5(28) \\
\text { - S-LANSS }<12-35.3(19)\end{array}$ & & \\
\hline \multirow[t]{5}{*}{$\begin{array}{l}\text { Brachial Plexus In } \\
\text { WADII/III } \\
(\mathrm{n}=416)\end{array}$} & $\begin{array}{l}\text { Sterling } 2002 \text { (WADII/ } \\
\text { III) } \\
\text { Chronic }\end{array}$ & $\begin{array}{l}\text { WAD -26.21 (95\%CI -28.05, -24.37), VAS } 4.93(4.6,5.3) \\
\text { Control -12.92 (95\%CI - 15.24, - 10.6), VAS } 2.62(2.2,3.04)\end{array}$ & \multirow[t]{5}{*}{4 case control } & \multirow[t]{5}{*}{$\begin{array}{l}\text { Very low: due to risk or bias, } \\
\text { directness }\end{array}$} \\
\hline & $\begin{array}{l}\text { Sterling 2003b (WADII/ } \\
\text { III) acute/sub-acute }\end{array}$ & $\begin{array}{l}<\mathbf{1} \text { months post injury [ROM mean (SD) (classified per } \\
\text { pain \& disability) } \\
\bullet \text { Recovered group (NDI }<8)-23.95 \text { (2.4) degrees, VAS } 1.8 \\
(04) \\
\bullet \text { Mild pain (NDI 10-28) -33.97 (2.6) degrees, VAS } 3.2 \\
(0.5) \\
\bullet \text { Moderate/severe (NDI >30) -34.27 (3.4) degrees, VAS } \\
4.1(0.5) \\
\bullet \text { Control -20.67 (3.12) degrees, VAS } 1.8(04) \\
\text { Mild (NDI } 10-28): \text { recovered and no different from } \\
\text { controls at } 2 \text { months }\end{array}$ & & \\
\hline & $\begin{array}{l}\text { Sterling 2003b (WADII/ } \\
\text { III) Chronic }\end{array}$ & $\begin{array}{l}6 \text { months } \\
\text { Moderate/severe (NDI >30): continued to have higher } \\
\text { VAS } 3.4(1.0) \text { and reduced elbow extension (no data } \\
\text { provided) }\end{array}$ & & \\
\hline & $\begin{array}{l}\text { Sterling } 2004 \text { (WADII/ } \\
\text { III) } \\
\text { Acute/sub-acute }\end{array}$ & $\begin{array}{l}\text { Mean (SD)degrees/VAS (pain \& disability) } \\
\text { - Mild (NDI 15.6) -26.7 (17.7), VAS 1.8 (1.7) } \\
\text { - Moderate (NDI 39.5) -31.3 (14.9), VAS 3.0 (1.8) } \\
\text { - Severe (NDI 69.5)-36.5 (11.8), VAS 4.3 (1.6) } \\
\text { - Control -21.4 (10.8), VAS 1.7 (1.7) }\end{array}$ & & \\
\hline & Chien 2009 (WADII) & $\begin{array}{l}\text { WAD -22.3 (27.4) degrees, VAS } 2.4(2.3) \\
\text { Control -11 (5.9), VAS } 0.7(1.1)\end{array}$ & & \\
\hline
\end{tabular}

BPI: brachial plexus irritation, BPPT: brachial plexus provocation test, NDI: Neck Disability Index, VAS: visual analogue scale, ROM: range of movement, S-LLANS: short version Leeds Assessment of Neuropathic Symptoms and Signs

Individuals with mild, moderate/severe presentations all share comparative levels of sternocleidomastoid activation in the acute [56] and chronic phases [12].

Myofascial pain and trigger points. Myofascial trigger points are highly prevalent in WAD with estimates ranging between $48-64 \%[16,37,40]$. Muscles that have been investigated include the middle/lower trapezius [16], scaleneus medius [40, 41] and sternocleidomastoid $[37,40,41]$, all with similar prevalence levels. Findings suggest that latent trigger points 
Table 8. Muscle dysfunction (muscle activation, pain and trigger points).

\begin{tabular}{|c|c|c|c|c|}
\hline Outcome & Studies & $\begin{array}{l}\text { Findings } \\
\text { Percentage (SD) change in } \\
\text { activation (EMG) unless stated }\end{array}$ & Grade & Overall GRADE \\
\hline \multirow[t]{5}{*}{ Muscle activation: all muscles- $(\mathrm{n}=220)$} & Klein 2001 & -no change & \multirow{5}{*}{$\begin{array}{l}4 \text { case control, } 1 \\
\text { single case study }\end{array}$} & \multirow{5}{*}{$\begin{array}{l}\text { Very low-due to risk or bias, } \\
\text { precision, consistency, directness }\end{array}$} \\
\hline & Sterling 2003a & $\begin{array}{l}\text { SCM (CCFT) } \\
\text { - Recovered 29(4), } \\
\text { - Mild 27(3), } \\
\text { - Moderate/severe 40(4), } \\
\text { - Control 16(3) }\end{array}$ & & \\
\hline & $\begin{array}{l}\text { Sterling } 2004 \\
\text { (acute) }\end{array}$ & $\begin{array}{l}\text { SCM (CCFT) } \\
\text { - Control 13(3), } \\
\text { - Mild 32 (3), } \\
\text { - Mod 29(4); } \\
\text { - Severe 47(4) }\end{array}$ & & \\
\hline & $\begin{array}{l}\text { Omar } 2007 \\
\text { (chronic WADIII) }\end{array}$ & -Weak mid/lower trapezius & & \\
\hline & Helgadottir 2011b & $\begin{array}{l}\text {-serratus anterior delayed onset, mid/ } \\
\text { low trapezius unchanged }\end{array}$ & & \\
\hline \multirow[t]{2}{*}{$\begin{array}{l}\text { Muscle activation: all muscle- Acute/sub- } \\
\text { acute }(\mathrm{n}=146)\end{array}$} & Sterling 2003a & $\begin{array}{l}\text { SCM (CCFT) } \\
\text { - Recovered } 29(4) \\
\text { - Mild } 27(3) \\
\text { - Moderate/severe } 40(4) \\
\text { Control } 16(3)\end{array}$ & \multirow[t]{2}{*}{2 case control } & \multirow[t]{2}{*}{ Very low-due to risk of bias } \\
\hline & Sterling 2004 & $\begin{array}{l}\text { - SCM (CCFT) } \\
\text { Control } 13 \text { (3), Mild } 32 \text { (3), Mod } 29 \\
\text { (4), Severe } 47 \text { (4) }\end{array}$ & & \\
\hline \multirow{3}{*}{$\begin{array}{l}\text { Muscle activation: all muscles- Chronic } \\
\text { WAD }(n=73)\end{array}$} & Klein 2001 & -no change & \multirow{3}{*}{$\begin{array}{l}2 \text { case control, } 1 \\
\text { single case study }\end{array}$} & \multirow{3}{*}{$\begin{array}{l}\text { Very low-due to risk or bias, } \\
\text { precision, consistency and directness }\end{array}$} \\
\hline & Omar 2007 & -Weak mid/lower trapezius & & \\
\hline & Helgadottir 2011b & $\begin{array}{l}\text {-serratus anterior delayed onset, mid } \\
\text { and lower trapezius unchanged }\end{array}$ & & \\
\hline \multirow{3}{*}{$\begin{array}{l}\text { Muscle activation: SCM (All stages) } \\
(\mathrm{n}=192)\end{array}$} & Klein 2001 & -no change & \multirow[t]{3}{*}{3 case control } & \multirow{3}{*}{$\begin{array}{l}\text { Very low-due to risk or bias, } \\
\text { precision, consistency and directness }\end{array}$} \\
\hline & Sterling 2003a & $\begin{array}{l}\text { SCM (CCFT) } \\
\text { - Recovered } 29 \text { (4), } \\
\text { - Mild } 27(3), \\
\text { - Moderate/severe } 40 \text { (4) }\end{array}$ & & \\
\hline & Sterling 2004 & $\begin{array}{l}\text { SCM (CCFT) } \\
\text { - Control 13 (3), } \\
\text { - Mild } 32(3), \\
\text { - Mod } 29(4), \\
\text { - Severe } 47(4) \\
\end{array}$ & & \\
\hline $\begin{array}{l}\text { Muscle activation: SCM- Sub-acute WAD } \\
\text { II/III }(\mathrm{n}=66)\end{array}$ & Sterling 2003a & $\begin{array}{l}\text { SCM (CCFT) } \\
\text { - Recovered } 29(4) \\
\text { - Mild } 27(3) \\
\text { - Moderate/severe } 40(4) \\
\text { - Control } 16(3)\end{array}$ & 1 case control & Very low-due to risk of bias \\
\hline \multirow[t]{2}{*}{$\begin{array}{l}\text { Muscle activation: SCM-Chronic WAD II/ } \\
\text { III }(\mathrm{n}=126)\end{array}$} & Klein 2001 & -no change & \multirow[t]{2}{*}{2 case control } & \multirow[t]{2}{*}{$\begin{array}{l}\text { Very low_due to risk or bias, } \\
\text { precision, consistency }\end{array}$} \\
\hline & Sterling 2004 & $\begin{array}{l}\text { SCM (CCFT) } \\
\text { - Control 13 (3) } \\
\text { - Mild } 32(3) \\
\text { - Mod } 29(4) \\
\text { - Severe } 47(4) \\
\end{array}$ & & \\
\hline $\begin{array}{l}\text { Myofascial pain and trigger points: all } \\
\text { muscles -Sub-acute WADII }(\mathrm{n}=20)\end{array}$ & $\begin{array}{l}\text { Fernandez-de-las- } \\
\text { Perez } 2012\end{array}$ & $\begin{array}{l}\text { Number of TPs } \\
\text { - WAD Scalene: Active R6, L4; } \\
\text { Latent R9, L10; No R5, L6 } \\
\text { - Control Scalene: Active R0, L0; } \\
\text { Latent R2, L4; No R18, L16 } \\
\text { - WAD SCM: Active R1, L6; Latent } \\
\text { R12, L9; No R7, L5 } \\
\text { - Control SCM: Active R0, L0; Latent } \\
\text { R4, L5; No R6, L15 }\end{array}$ & 1 case control & Very low-due to risk or bias \\
\hline
\end{tabular}


Table 8. (Continued)

\begin{tabular}{|c|c|c|c|c|}
\hline Outcome & Studies & $\begin{array}{l}\text { Findings } \\
\text { Percentage (SD) change in } \\
\text { activation (EMG) unless stated }\end{array}$ & Grade & Overall GRADE \\
\hline \multirow[t]{3}{*}{$\begin{array}{l}\text { Myofascial pain and trigger points: all } \\
\text { muscles-Chronic } \operatorname{WAD}(n=121)\end{array}$} & $\begin{array}{l}\text { Bismil } 2005 \\
\text { (WADII) }\end{array}$ & Prevalence: $48 \%$ & \multirow[t]{3}{*}{$\begin{array}{l}2 \text { case control, } 1 \\
\text { cohort }\end{array}$} & \multirow[t]{3}{*}{$\begin{array}{l}\text { Very low-due to risk or bias, } \\
\text { reporting bias, directness }\end{array}$} \\
\hline & Ettlin 2008 & $\begin{array}{l}\text { Prevalence: } \\
\text { - WAD: Scaleneus medius } 63.8 \% \text {, } \\
\text { SCM } 51.1 \% \\
\text { - Control: Scaleneus medius } 12.5 \% \text {, } \\
\text { SCM } 12.5 \%\end{array}$ & & \\
\hline & $\begin{array}{l}\text { Castaldo } 2014 \\
\text { (WADII \& III) }\end{array}$ & $\begin{array}{l}\text { Number of TPs (SCM) } \\
\text { - WAD: Active L 21, R 22; Latent L } \\
\text { 13, R } 15 \\
\text { - Control (MNP): Active L 13, R 13; } \\
\text { Latent L 6, R } 9\end{array}$ & & \\
\hline $\begin{array}{l}\text { Myofascial pain: Trapezius (mid/low) } \\
\text { Chronic WADII }(\mathrm{n}=25)\end{array}$ & Bismil 2005 & Prevalence: 48\% & 1 cohort & $\begin{array}{l}\text { Very low-due to risk or bias, } \\
\text { precision, reporting bias, directness }\end{array}$ \\
\hline \multirow[t]{3}{*}{$\begin{array}{l}\text { Myofascial pain and trigger points: SCM- } \\
\text { Chronic and sub-acute WAD }(n=116)\end{array}$} & Ettlin 2008 & $\begin{array}{l}\text { Prevalence } \\
\text { - WAD: } 51.1 \% \\
\text { - Control: } 12.5 \% \\
\end{array}$ & \multirow[t]{3}{*}{3 case control } & \multirow[t]{3}{*}{$\begin{array}{l}\text { Very low-due to risk or bias, } \\
\text { precision (?), reporting bias, } \\
\text { directness }\end{array}$} \\
\hline & $\begin{array}{l}\text { Fernandez-de-las- } \\
\text { Perez } 2012\end{array}$ & $\begin{array}{l}\text { Number of TPs } \\
\text { - WAD: Active R1, L6; Latent R12, } \\
\text { L9; No R7, L5 } \\
\text { • Control: Active R0, L0; Latent R4, } \\
\text { L5; No R 6, L15 }\end{array}$ & & \\
\hline & Castaldo 2014 & $\begin{array}{l}\text { Number of TPs } \\
\text { - WAD: Active L 21, R 22; Latent L } \\
\text { 13, R 15 } \\
\text { - Control (MNP): Active L13, R 13; } \\
\text { Latent MTP L 6, R } 9\end{array}$ & & \\
\hline \multirow[t]{2}{*}{$\begin{array}{l}\text { Myofascial pain and trigger points: } \\
\text { Scalene- } \\
\text { Chronic WAD and sub-acute }(n=67)\end{array}$} & Ettlin 2008 & $\begin{array}{l}\text { Prevalence } \\
\text { - WAD: } 63.8 \% \\
\text { - Control: } 12.5 \%\end{array}$ & \multirow[t]{2}{*}{2 case control } & \multirow[t]{2}{*}{$\begin{array}{l}\text { Very low_due to risk or bias, } \\
\text { precision, directness }\end{array}$} \\
\hline & $\begin{array}{l}\text { Fernandez-de-las- } \\
\text { Perez } 2012\end{array}$ & $\begin{array}{l}\text { Number of TPs } \\
\text { - WAD: Active R 6, L4; Latent R 9, } \\
\text { L10; No R 5, L6 } \\
\text { • Control: Active R0, L0; Latent R2, } \\
\text { L4; No R 18, L16 }\end{array}$ & & \\
\hline
\end{tabular}

SCM: sternocleidomastoid, CCFT: craniocervical flexion test, MTP: myofascial trigger points, MNP: mechanical neck pain

are more prevalent in sub-acute WADII [41] whereas in chronic WAD there is a higher prevalence of active trigger points [37]. It should however be noted that the sample of chronic WAD comprised both WADII and III, so perhaps severity could partly explain the differences seen. Likewise, age may account for some of the differences given the sub-acute sample was 28.7 years [41] compared 41.6 years in the chronic WAD group [37].

\section{Discussion}

\section{Summary of evidence}

This is the first methodologically rigorous systematic review investigating thoracic dysfunction in whiplash associated disorders. From a comprehensive search, 38 studies were included and evaluated as part of the review. Many studies were at risk of bias, primarily due to poor reporting with most studies published prior to the introduction of the STROBE reporting guidelines 
for observational studies. Notwithstanding the low quality of the evidence, there is unequivocal evidence of thoracic dysfunction in WAD.

Pain. Although there is a high prevalence of acute and chronic pain experienced in the thoracic spine region following injury, distinguishing the interplay of pain mechanisms is however challenging. Whilst we know peripheral and central sensitisation begin immediately following injury [64], it is plausible that damage to thoracic musculoskeletal tissues contributes to the relatively high prevalence of pain reported in the acute/sub-acute stages from peripheral nociceptor stimuli [11, 14, 17, 43, 52]. Relatively few studies in this review reported perceived pain levels e.g. VAS, making it difficult to consider an association between injury severity and tissue damage. Although reviewed in detail by Van Oosterwijck et al, [64] the current review found just one study reporting thoracic pain specifically associated with central sensitisation, with allodynia reported in $71 \%$ of individuals with CWADI/II [33], arguably contributing to the lack of consistency with findings for chronic WAD where differing pain mechanisms may co-exist. Likewise distinguishing local from referred pain is challenging where injury in the cervical spine may refer pain caudally to the upper and mid thoracic region [65]. Unlike the thoracic spine, reported chest pain prevalence was relatively low, perhaps more closely associated with severe injuries, including fractures, which were excluded from this review. Future studies should, in addition to using the QTF Classification, include self-reported pain severity for each anatomical region.

Thoracic posture and mobility. With limited very low quality evidence and relative to the cervical spine, there is a paucity of research investigating thoracic posture and mobility in WAD $[44,45,48,61]$. This may be explained with priority being given to areas with most severe pain, with the cervical spine and associated tissues being most vulnerable to stress and damage compared to the relatively stable and stiff thoracic spine [21], arguably enhanced with the mandatory use of seatbelts offering additional stability. However, considering the effect of a forceful injury on posterior structures, and neck stiffness being a hallmark of chronic WAD further research is required, specifically to investigate thoracic posture, mobility and muscle stiffness in WAD, all of which may offer new directions for research into management of WAD.

Thoracic outlet syndrome. Although this review suggests a relatively high prevalence of thoracic outlet syndrome, the quality of evidence is very low and derived from relatively older studies, including two studies from the same group of authors [35,36]. Whilst the mechanism of injury and resultant strain on the scalene triangle in WAD would, in theory, place the thoracic outlet at risk of injury, this does needs to be investigated further. Likewise as a 'syndrome', this does not provide primary evidence of a primary structural dysfunction; it merely provides evidence of a dysfunction which, in turn, could be neurogenic, myogenic, vasculogenic in nature. Perhaps, in the absence of evidence of thoracic outlet syndrome in acute $\mathrm{WAD}$, this condition is secondary to the consequences of whiplash, altered posture, changes in muscle behaviour etc. [66]. Future studies should use robust observational study designs and include valid assessment techniques to diagnose thoracic outlet syndrome.

Brachial plexus. There is considerable evidence of brachial plexus dysfunction in both acute/sub-acute and chronic WAD from research investigating the brachial plexus provocation test. Although coming from a relatively small group of researchers, the evidence supports further investigation. Future research could usefully consider the relationship of brachial plexus dysfunction to other musculoskeletal changes in the cervical and thoracic spine following a whiplash injury, but also approaches to managing this; a notable gap within the whiplash management evidence $[9,10,66]$. Although inclusion of the brachial plexus in this review may at first appear tenuous, with contributions from the level of $\mathrm{T} 1$ and its relationship to the thoracic outlet, its inclusion provides good evidence to further explore this anatomically and 
functionally challenging cervico-thoracic-supraclavicular region; a transitional zone between the stable/stiff thoracic spine and relatively mobile cervical spine.

Muscle activation. There is unequivocal evidence of altered function of muscles following whiplash injury [66], however this evidence is largely limited to cervical muscles, with this review identifying just three muscles with insertions to the thoracic region, sternocleidomastoid [12, 49, 56]; serratus anterior [45]; middle and lower fibres of trapezius [45, 54]. Although it is difficult to derive meaningful conclusions with so few muscles investigated and the quality of the evidence, the observed positive relationship between sternocleidomastoid activation and pain severity, and evidence across all stages post injury $[12,49,56]$ supports the need for further research into altered activation of cervico-thoracic and thoracic muscles, ideally involving functional spinal movements; something that is now feasible with advances in technology, including high density EMG.

Myofascial pain and trigger points. This review has identified that myofascial trigger points are highly prevalent in WAD $[16,37,40]$, although again from very low quality evidence and limited to a small number of muscles; middle/lower trapezius [16], scaleneus medius [40, 41] and sternocleidomastoid [37, 40, 41]. Nonetheless all muscles have similar prevalence levels of trigger points, with a higher prevalence of latent trigger points in sub-acute WAD [41] and active trigger points in chronic WAD [37]. With evidence of trigger points across all muscles and across the stages and severity of presentations, research is now required to explore other muscles in the thoracic region and better understand the development of pain, and persistent pain seen in chronic WAD, perhaps with longitudinal studies. Notwithstanding the quality, evidence was found of myofascial-entheseal dysfunction [16], a relatively new clinical entity and arguably similar to insertional tendinopathies. With rapid advances in our understanding of injury induced tendinopathies in the lower limb, this does offer new insights to possible muscle pathologies which may contribute to persistent pain and disability seen in WAD.

This review provides unequivocal evidence of thoracic dysfunction in WAD, albeit from evidence of low/very low quality. The findings do support a more extensive clinical evaluation of patients following a whiplash injury and the need for more methodologically robust observational studies to further characterise thoracic dysfunction in WAD across stages of the condition and levels of severity. Knowledge and understanding of thoracic dysfunction, where anatomical and biomechanical relationships with the cervical spine exist, offers novel directions for research into management of this disabling condition.

Research into WAD management has been, and continues to be primarily focused on managing cervical spine dysfunction, with interventions such as manual therapy, exercise etc. targeting the cervical spine [66], and targeting the psychological impact of a whiplash injury [66]. With inconclusive evidence of the therapeutic value of the above [67] it is perhaps time to consider new directions for research.

Whilst exercise as part of multimodal packages of care is recommended in the management of acute and chronic WAD $[66,68]$, the range of approaches available are considerable e.g. graded functional exercise, postural exercises, and strengthening and motor control exercises etc.[66]. The authors are not however aware of any recommendations or research specifically supporting the inclusion of thoracic spine exercises, although these could reasonably be incorporated within functional exercise programmes. With at best short term modest improvements in current exercise interventions $[9,10]$ and some evidence of some therapeutic value of thoracic spine manipulation in WAD [69] further research to investigate interventions targeting the thoracic spine and related dysfunction is justified.

It has not until recent years that the thoracic spine, coined the 'Cinderella' of the spine [21], has started to receive more research interest. This has been attributed partly to the relatively 
lower prevalence of symptoms than the cervical and lumbar spine more generally, but also due to the lack of affordable, non-invasive and valid measurement tools to evaluate motion in this anatomically complex and relatively stiff spinal region [21]. With the development of new measurement approaches for thoracic posture and mobility $[63,70,71]$ we now have tools to support research of thoracic posture and mobility in the thoracic spine region in painful neck disorders.

\section{Strengths and limitations of this review}

This review is rigorous and original, with a design and focus on the thoracic spine region using a pre-defined rigorous and published protocol with subject and methodological experts contributing to the evaluation. The key limitation of the review is lack of high quality evidence and compounding this was that few authors responded to requests for additional information or data.

\section{Conclusions}

This first and rigorous systematic review found considerable evidence of thoracic pain and dysfunction in patients at all stages following whiplash injury. Notwithstanding the low/very low level of evidence, our findings do support a more extensive clinical evaluation of patients presenting with WAD. Key findings include 1) a high prevalence of thoracic spine pain, with the highest levels of pain immediately following injury and in more severe presentations 2) evidence of muscle dysfunction (delayed onset or heightened levels of activity) in a limited number of muscles 3 ) evidence of thoracic outlet syndrome and brachial plexus involvement 4) inconclusive/limited evidence of postural changes and effect on thoracic spinal mobility. Additional high quality research is required to further characterise dysfunction across other structures in the thoracic region, including but not limited to the thoracic spine (mobility and posture) and thoracic muscles (stiffness, activation patterns). In turn this may inform the design of clinical trials targeting such dysfunction.

\section{Supporting information}

S1 Table. PRISMA 2009 checklist.

(DOC)

S2 Table. Medline search.

(DOCX)

S3 Table. Excluded papers and reasons.

(DOCX)

\section{Author Contributions}

Conceptualization: Nicola R. Heneghan, Alison Rushton.

Data curation: Nicola R. Heneghan, Richard Smith, Isaak Tyros, Alison Rushton.

Formal analysis: Nicola R. Heneghan, Richard Smith, Isaak Tyros, Deborah Falla, Alison Rushton.

Investigation: Nicola R. Heneghan.

Methodology: Nicola R. Heneghan, Richard Smith, Alison Rushton.

Project administration: Nicola R. Heneghan. 
Validation: Nicola R. Heneghan, Richard Smith, Isaak Tyros, Deborah Falla, Alison Rushton.

Visualization: Nicola R. Heneghan, Richard Smith, Isaak Tyros, Deborah Falla, Alison Rushton.

Writing - original draft: Nicola R. Heneghan, Richard Smith, Isaak Tyros, Deborah Falla, Alison Rushton.

Writing - review \& editing: Nicola R. Heneghan, Richard Smith, Isaak Tyros, Deborah Falla, Alison Rushton.

\section{References}

1. Holm LW CL, Cassidy JD, Hogg-Johnson S, Côté P, Guzman J, Peloso P, et al. The burden and determinants of neck pain in Whiplash associated disorders after traffic collisions, results of the Bone and Joint Decade 2000-2010 Task Force on Neck pain and its Associated Disorders. Spine. 2008; 33(4 Suppl):S52-9. https://doi.org/10.1097/BRS.0b013e3181643ece PMID: 18204401

2. Johansson MS BE, Hartvigsen J, Stochkendahl MJ, Carroll L, Cassidy JD A population-based, incidence cohort stuidy of mid-back pain after traffic collisions: Factors associated with global recovery. Eur J of Pain. 2015; 19(10):1486-95

3. Spitzer WO SM, Salmi LR, Cassidy JD, Duranceau J, Suissa S, Zeiss E. Scientific monograph of the Quebec Task Force on Whiplash Associated Disorders: redefining 'whiplash' and its management. Spine. 1995; 20(8 Suppl):1S-73S. PMID: 7604354

4. Walton DM MJ, Giorgianni AA, Mascarenhas JC, West SC, Zammit CA Risk factors for persistent problems following acute whiplash injury: Update of a systematic review and meta-analysis. JOSPT. 2013; 43(2):31-43. https://doi.org/10.2519/jospt.2013.4507 PMID: 23322093

5. Williamson E WM, Gates S, Lamb SE. Risk factors for chronic disability in a cohort of patients with acute whiplash associated disorders seeking physiotherapy treatment for persisting symptoms Physiotherapy. 2014; 101(1):34-43. https://doi.org/10.1016/j.physio.2014.04.004 PMID: 24996567

6. Chien A SM. Sensory hypoaesthesia is a feature of chronic whiplash but not chronic idiopathic neck pain. Man Ther. 2010; 15:48-53. https://doi.org/10.1016/j.math.2009.05.012 PMID: 19632884

7. Eck JC HS, Humphreys SC. Whiplash: a review of a commonly misunderstood injury. American Journal of Medicine. 2001; 110:651-6. PMID: 11382374

8. Galasko CSB MP, Stephenson W. Incidence of whiplash-associated disorder. BC Medical Journal. 2002; 44:237-40.

9. Rushton A WC, Heneghan NR, Eveleigh G, Calvert M, Freemantle N. Physiotherapy rehabilitation for Whiplash Associated Disorder II: a systematic review and meta-analysis of Randomised Controlled Trials. BMJ Open. 201114 ( 1(2):e000265). https://doi.org/10.1136/bmjopen-2011-000265 PMID: 22102642

10. Wiangkham T DJ, Haque S, Madi M, Rushton M. The Effectiveness of Conservative Management for Acute Whiplash Associated Disorder (WAD) II: A Systematic Review and Meta-Analysis of Randomised Controlled Trials. PLoS ONE [Electronic Resource]. 2015; 10 (7):e0133415.

11. Bortsov AV, Platts-Mills TF, Peak DA, Jones JS, Swor RA, Domeier RM, et al. Effect of pain location and duration on life function in the year after motor vehicle collision. Pain management. 2014; 155 (9):1836-45.

12. Sterling M, Jull G, Vicenzino B, Kenardy J. A proposed new classification system for whiplash associated disorders-implications for assessment and management. Man Ther. 2004; 9:60-70. https://doi. org/10.1016/j.math.2004.01.006 PMID: 15040964

13. Woodhouse A V. Altered motor control patterns in whiplash and chronic neck pain. BMC Musculoskeletal Disorder. 2008; 9(90)

14. Hincapié CA, Cassidy JD, Côté P, Carroll LJ, Guzmán J. Whiplash Injury is More Than Neck Pain: A Population-Based Study of Pain Localization After Traffic Injury. Journal of Occupational and Environmental Medicine. 2010; 52(4):434-40. https://doi.org/10.1097/JOM.0b013e3181bb806d 00043764201004000-00011. PMID: 20357684

15. Vasavada AN BJ, Siegmund GP Musculotendon and Fasicle Strains in Anterior and Posterior Neck Muscle During Whiplash Injury. Spine. 2007; 32(7):756-65 https://doi.org/10.1097/01.brs.0000259058. 00460.69 PMID: 17414909

16. Bismil Q, Bismil M. Myofascial-entheseal dysfunction in chronic whiplash injury: an observational study. JRSM short reports. 2012; 3(8):57. https://doi.org/10.1258/shorts.2012.012052 PMID: 23301145. 
17. Holm LW CL, Cassidy JD, Skillgate E, Ahlbom A. Widespread pain following whiplash-associated disorders: incidence, course, and risk factors. Journal of Rheumatology. 2007; 34(1):193-200. PMID: 17143964

18. Gerdle B, Hilgenfeldt U, Larsson B, Kristiansen J, Sogaard K, Rosendal L. Bradykinin and kallidin levels in the trapezius muscle in patients with work-related trapezius myalgia, in patients with whiplash associated pain, and in healthy controls-A microdialysis study of women. Pain. 2008; 139(3):578-87. https:// doi.org/10.1016/j.pain.2008.06.012 PMID: 18657364.

19. Nederhand MJ HH, IJzerman NJ, Turk DC, Zivold G Cervical muscle dysfunction in chronic whiplashassociated disorder grade 2: the relevance of the trauma. Spine. 2002; 27(10):1056-61. PMID: 12004172

20. Tsang SM, Szeto GP, Lee RY. Normal kinematics of the neck: the interplay between the cervical and thoracic spines. Man Ther. 2013; 18(5):431-7. https://doi.org/10.1016/j.math.2013.03.002 PMID: 23632368

21. Heneghan NR, Rushton A. Understanding why the thoracic region is the 'Cinderella' region of the spine. Man Ther. 2016; 21:274-6. https://doi.org/10.1016/j.math.2015.06.010 PMID: 26189592

22. Heneghan NR, Smith R, Rushton A. Thoracic dysfunction in whiplash-associated disorders: a systematic review and metaanalysis protocol. Systematic Reviews. 2016;(26):5.

23. Dissemination CfRa. Systematic Reviews: CRD's Guidance for Undertaking Reviews in Healthcare. CRD University of York, York Publishing Services Ltd. 2009.

24. Stroup DF BJ, Morton SC, Olkin I, Williamson GD, Rennie D, Moher D, et al. Meta-analysis of Observational Studies in Epidemiology A Proposal for Reporting. JAMA. 2000; 283(15):2008-12. PMID: 10789670

25. Moher D LA, Tetzlaff J, Altman DG for the PRISMA Group. Preferred reporting items for systematic reviews and meta-analyses: the PRISMA statement. BMJ. 2009;(339):b2535.

26. Cooke A, Smith D. \& Booth A. Beyond PICO: the SPIDER tool for qualitative evidence sythesis. Qualitative Health Research. 2012; 22(10):1435-43. https://doi.org/10.1177/1049732312452938 PMID: 22829486

27. van Tulder M FA, Bombardier $C$. Bouter $L$ and the Editorial Board of the Cochrane Collaboration back review group. Updated method guidelines for systematic reviews in the Cochrane Collaboration back review group. Spine. 2003; 28(1290e9).

28. Furlan A PV, Bombardier C,. Updated method guidelines for systematic reviews in the Cochrane Collaboration back review group. Spine. 2009; 34(1929e41).

29. Deeks JJ DJ, D'Amico R. Evaluating non-randomised intervention studies. Health Technol Assess 2003; 7:iii-X,:1-173.

30. Guyatt GH OA, Vist G, Kunz R, Falck-Ytter Y, Alonso-Coello P, Schünemann HJ. GRADE: an emerging consensus on rating quality of evidence and strength of recommendations. BMJ 2008;(336):924-6.

31. Alexandre A, Coro L, Azuelos A, Pellone M. Thoracic outlet syndrome due to hyperextension-hyperflexion cervical injury. Act Neur S. 2005; 92:21-4. WOS:000228254500005.

32. Berglund A, Alfredsson L, Jensen I, Cassidy J, David Nygren A. The association between exposure to a rear-end collision and future health complaints. J Clin Epidemiol. 2001; 54(8):851-6. http://dx.doi.org/ 10.1016/S0895-4356\%2800\%2900369-3. PMID: 11470396.

33. Bock SL, Centeno CJ, Elliott JM. The presence and interrater reliability of thoracic allodynia in a whiplash cohort. Pain Physician. 2005; 8(3):267-70. PMID: 16850083.

34. Bodack. Spinal Accessory Nerve Palsy as a Cause of Pain After Whiplash Injury: Case Report. 1998.

35. Capistrant TD. Thoracic outlet syndrome in whiplash injury. Ann Surg. 1977; 185(2):175-8. Epub 1977/ 02/01. PMID: 836089; PubMed Central PMCID: PMCPmc1396108.

36. Capistrant TD. Thoracic outlet syndrome in cervical strain injury. Minnesota medicine. 1986; 69(1):137. Epub 1986/01/01. PMID: 3951417.

37. Castaldo M, Ge HY, Chiarotto A, Villafane $\mathrm{JH}$, Arendt-Nielsen L. Myofascial trigger points in patients with whiplash-associated disorders and mechanical neck pain. Pain Med. 2014; 15(5):842-9. https:// doi.org/10.1111/pme.12429 PMID: 24641263.

38. Chien A EL, Sterling M. Hypoaesthesia occurs with sensory hypersensitivity in chronic whiplash e Further evidence of a neuropathic condition. Man Ther. 2009.

39. Cornips E. Crippling upper back pain after whiplash and other motor vehicle collisions caused by thoracic disc herniations: Report of 10 cases. Spine. 2014; 39(12):988-95. http://dx.doi.org/10.1097/BRS. 0000000000000332 . PMID: 24718062.

40. Ettlin T, Schuster C, Stoffel R, Brudrlin A, Kischka U. A Distinct Pattern of Myofascial Findings in Patients After Whiplash Injury. Archives of Physical Medicine \& Rehabilitation 2008; 89:1290-4. 
41. Fernandez-Perez AM, Villaverde-Gutierrez C, Mora-Sanchez A, Alonso-Blanco C, Sterling M, Fernandez-de-Las-Penas $C$. Muscle trigger points, pressure pain threshold, and cervical range of motion in patients with high level of disability related to acute whiplash injury. The Journal of orthopaedic and sports physical therapy. 2012; 42(7):634-41. https://doi.org/10.2519/jospt.2012.4117 PMID: 22677576.

42. Ferrari R. Predicting central sensitisation Whiplash patients. Australian Family Physician. 2010.

43. Hartling L PW, Brison RJ. Derivation of a clinical decision rule for whiplash associateddisorders among individuals involved in rear-end collisions Accident Anal Prev. 2002; 34:531-9.

44. Helgadottir $\mathrm{H}$, Kristjansson $\mathrm{E}$, Mottram $\mathrm{S}$, Karduna A, Jonsson $\mathrm{H}$ Jr. Altered alignment of the shoulder girdle and cervical spine in patients with insidious onset neck pain and whiplash-associated disorder. Journal of Applied Biomechanics. 2011a; 27(3):181-91. PMID: 2011430451.

45. Helgadottir $\mathrm{H}$, Kristjansson E, Einarsson E, Karduna A, Jonsson H Jr. Altered activity of the serratus anterior during unilateral arm elevation in patients with cervical disorders. Journal of electromyography and kinesiology: official journal of the International Society of Electrophysiological Kinesiology. 2011b; 21(6):947-53. https://doi.org/10.1016/j.jelekin.2011.07.007 PMID: 21889362.

46. Ide MJI, M. Yamaga, K. Takagi. Symptoms and signs of irritation of the brachial plexus in whiplash injuries. 2001.

47. Kai $Y$, Oyama M, Kurose $S$, Inadome $T$, Oketani $Y$, Masuda $Y$. Neurogenic thoracic outlet syndrome in whiplash injury. Journal of spinal disorders. 2001; 14(6):487-93. https://doi.org/10.1097/00002517200112000-00004 WOS:000172596700004. PMID: 11723397

48. Kall LB. Assessment of motion in the cervico-thoracic spine in patients with subacute whiplash-associated disorders. Journal of Rehabiliation Med. 2008; 40:418-25.

49. Klein GN MA, Panjabi MM, Dvorak J. Trapped in the neutral zone: another symptom of whiplash-associated disorder? 2001.

50. Koelbaek Johansen M, Graven-Nielsen T, Schou Olesen A, Arendt-Nielsen L. Generalised muscular hyperalgesia in chronic whiplash syndrome. Pain. 1999; 83(2):229-34. PMID: 10534594.

51. Magnusson T. Extracervical Symptoms after Whiplash Trauma. Cephalalgia: an international journal of headache. 1994; 14(3):223-37. https://doi.org/10.1046/j.1468-2982.1994.014003223.x WOS: A1994NX52400014. PMID: 7954744

52. McLean SA, Ulirsch JC, Slade GD, Soward AC, Swor RA, Peak DA, et al. Incidence and predictors of neck and widespread pain after motor vehicle collision among US litigants and nonlitigants. Pain. 2014; 155(2):309-21. https://doi.org/10.1016/j.pain.2013.10.016 PMID: 24145211; PubMed Central PMCID: PMCNIHMS546545

53. Myran R HK, Svebak S, Nygaard O, Zwart JA. Headache and musculoskeletal complaints among subjects with self reported whiplash injury. The HUNT-2 study. BMC Musculoskeletal Disorder. 2011; 12:129-.

54. Omar N, Alvi F, Srinivasan M. An unusual presentation of whiplash injury: Long thoracic and spinal accessory nerve injury. European Spine Journal. 2007; 16(SUPPL. 3):S275-S7. http://dx.doi.org/10. 1007/s00586-007-0413-z. PMID: 2007624656.

55. Sterling M TJ, Jull G. Responses to a clinical test of mechanical provocation of nerve tissue in whiplash associated disorder. Man Ther. 2002.

56. Sterling M J, Vicenzino B, Kenardy J, Darnell R. Development of motor system dysfunction following whiplash injury. Pain. 2003a.

57. Sterling M JG, Vicenzino B, Kenardy J. Sensory hypersensitivity occurs soon after whiplash injury and is associated with poor recovery. Pain. 2003b; 104:509-17.

58. Sterling M JG, Vicenzino B, Kenardy J, Darnell R. Physical and psychological factors predict outcome following whiplash injury. Pain management. 2005; 114:141-8.

59. Sterling M PA. A neuropathic pain component is common in acute whiplash and associated with a more complex clinical presentation. Man Ther. 2009; 14:173-9. https://doi.org/10.1016/j.math.2008.01.009 PMID: 18358761

60. Wenzel HG MA, Nilsen TI. Symptom profile of persons self-reporting whiplash: a Norwegian population-based study (HUNT 2). Eur Spine J. 2009; 18(9):1363-70. https://doi.org/10.1007/s00586-0091106-6 PMID: 19669172

61. Wirth B, Amstalden M, Perk M, Boutellier U, Humphreys BK. Respiratory dysfunction in patients with chronic neck pain —Influence of thoracic spine and chest mobility. Man Ther. 2014; 19(5):440-4. https://doi.org/10.1016/j.math.2014.04.011 WOS:000347251900013. PMID: 24835338

62. Yeung $\mathrm{E}$, Jones $\mathrm{M}, \mathrm{Hall} \mathrm{B}$. The response to the slump te in a group of female whiplash patients. Australian Journal of Physiotherapy. 1997; 43(4):245-52. PMID: 11676694 
63. Barrett MK, Lewis J. Reliability and validity of non-radiographic methods of thoracic kyphosis measurement: A systematic review. Man Ther. 2014; 19:10-7. https://doi.org/10.1016/j.math.2013.09.003 PMID: 24246907

64. Van Oosterwijck J NJ, Meeus M, Paul L. Evidence for central sensitization in chronic whiplash:A systematic literature review. Eur J of Pain. 2013;(17):299-312.

65. Louw A SS. Chronic pain and the thoracic spine. J Man Manip Ther. 2015; 23(3):162-8. https://doi.org/ 10.1179/2042618615Y.0000000006 PMID: 26308707

66. Michelle S. Physiotherapy management of whiplash-associated disorders (WAD). Journal of Physiotherapy 2014;(60):5-12.

67. Shearer HM CL, Wong JJ, Côté $P$, Varatharajan S, Southerst D, Sutton DA, et al Are psychological interventions effective for the management of neck pain and whiplash-associated disorders? A systematic review by the Ontario Protocol for Traffic Injury Management (OPTIMa) Collaboration Spine. 2015.

68. Sutton DA Cote CP, Wong JJ, Varatharajan S, Randhawa KA, Yu H, et al. Is multimodal care effective for the management of patients with whiplash-associated disorders or neck pain and associated disorders? A systematic review by the Ontario Protocol for Traffic Injury Management (OPTIMa) Collaboration. Spine. 2014;S1529-9430(14):00650-0.

69. Fernandez-de-las-Penas C F-CJ, Fernandez AP, Lomas-Varga R, Miangolarra-Page JC. Dorsal manipulation in whiplash injury treatment: A randomized controlled trial. J Whiplash Rel Disord. 2004; 3:55-72.

70. Heneghan NR, Hall A, Hollands M, Balanos GM. Stability and intra-tester reliability of an in vivo measurement of thoracic axial rotation using an innovative methodology. Man Ther. 2009; 14(4):452-5 https://doi.org/10.1016/j.math.2008.10.004 PMID: 19046655

71. Bucke J, Spencer S, Fawcett L, Sonvico L, Rushton A, Heneghan NR. Validity of the digital inclinometer and iPhone when measuring thoracic rotation. Journal of Athletic Training. 2017; 52 (9):820-5. https:// doi.org/10.4085/1062-6050-52.6.05 PMID: 28787176 